\title{
Faster speciation of fig-wasps than their host figs leads to decoupled speciation 1 dynamics: snapshots across the speciation continuum
}

by Souto-Vilaros, D., Machac, A., Michalek, J., Darwell, C.T., Sisol, M., Kuyaiva, B.I., Weiblen, G.D., Novotny, V. and Segar, S.T.

Copyright, publisher and additional information: this is the authors accepted manuscript. The final published version (version of record) is available online via Wiley. This article may be used for non-commercial purposes in accordance with Wiley Terms and Conditions for Self-Archiving.

Please refer to any applicable terms of use of the publisher.

DOI: $\underline{\text { https://doi.org/10.1111/mec.15190 }}$

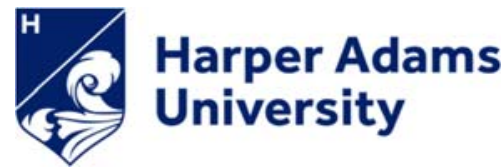

Souto-Vilaros, D., Machac, A., Michalek, J., Darwell, C.T., Sisol, M., Kuyaiva, B.I., Weiblen, G.D., Novotny, V. and Segar, S.T. 2019. Faster speciation of fig-wasps than their host figs leads to decoupled speciation 1 dynamics: snapshots across the speciation continuum. Molecular Ecology. 
1 Faster speciation of fig-wasps than their host figs leads to decoupled speciation

2 dynamics: snapshots across the speciation continuum

3 Daniel Souto-Vilarós ${ }^{1,2}$, Antonin Machac ${ }^{3-6}$, Jan Michalek ${ }^{2}$, Clive T. Darwell ${ }^{7}$, Mentap Sisol ${ }^{8}$, 4 Thomas Kuyaiva ${ }^{8}$, Brus Isua $^{8}$, George D. Weiblen ${ }^{9}$, Vojtech Novotny ${ }^{2,8}$, Simon T. Segar ${ }^{2,10}$

$6{ }^{1}$ Faculty of Science, University of South Bohemia, Branisovska 1645/31A, 370 05, South

7 Bohemia, Czech Republic

$8{ }^{2}$ Biology Centre of the Czech Academy of Sciences, Institute of Entomology, Branisovska 9 1645/31A, 370 05, South Bohemia, Czech Republic

$10{ }^{3}$ Center for Theoretical Study, Charles University and Czech Academy of Sciences, Jilska 1, 11000, Prague, Czech Republic

${ }^{4}$ Department of Ecology, Charles University, Vinicna 7, 12844 Prague, Czech Republic

${ }^{5}$ Center for Macroecology, Evolution and Climate, Natural History Museum of Denmark,

University of Copenhagen, DK-2100 Copenhagen, Denmark

${ }^{6}$ Biodiversity Research Centre, University of British Columbia, 2212 Main Mall, Vancouver

V6T 1Z4, Canada

${ }^{7}$ Okinawa Institute of Science and Technology Graduate University, 1919-1 Tancha, Onnason, Kunigami-gun, Okinawa, Japan

${ }^{8}$ New Guinea Binatang Research Centre, Nagada Harbour, Madang, Papua New Guinea

${ }^{9}$ Institute on the Environment, University of Minnesota, 1954 Buford Ave., Saint Paul, Minnesota 55108, U.S.A.

${ }^{10}$ Department of Crop and Environment Sciences, Harper Adams University, Newport, TF10 8NB, United Kingdom 
Abstract

Even though speciation involving multiple interacting partners, such as plants and their pollinators, has attracted much research, most studies focus on isolated phases of the process. This currently precludes an integrated understanding of the mechanisms leading to cospeciation. Here, we examine population genetic structure across six species-pairs of figs and their pollinating wasps along an elevational gradient in New Guinea. Specifically, we test three hypotheses on the genetic structure within the examined species-pairs and find that the hypothesized genetic structures represent different phases of a single continuum, from incipient co-speciation to the full formation of new species. Our results also illuminate the mechanisms governing co-speciation, namely that fig wasps tend to accumulate population genetic differences faster than their figs, which initially decouples the speciation dynamics between the two interacting partners and breaks down their one-to-one matching. This intermediate phase is followed by genetic divergence of both partners, which may eventually restore the one-to-one matching among the fully formed species. Together, these findings integrate current knowledge on the mechanisms operating during different phases of the cospeciation process. They also reveal that the increasingly reported breakdowns in one-to-one matching may be an inherent part of the co-speciation process. Mechanistic understanding of this process is needed to explain how the extraordinary diversity of species, especially in the tropics, has emerged. Knowing which breakdowns in species interactions are a natural phase of co-speciation and which may endanger further generation of diversity seems critical in a constantly changing world.

Keywords: Fig and fig-wasp mutualism, population genomics, population structure, cospeciation, altitudinal gradient, Papua New Guinea 
How species originate has been one of the central questions in biology, and we still only have an incomplete answer, especially when the process involves interactions between multiple species. These interactions both generate and maintain biodiversity, where interacting species complexes promote or constrain diversification of each other (Forister \& Feldman, 2011). Some of the most suitable systems for research are those following tight one-to-one matching, such as those between some plants and their pollinators. Among the most widely studied, is the mutualistic interaction between figs (genus Ficus, Moraceae) and their pollinating fig-wasps (Agaonidae) (Cruaud et al., 2012; Kiester, Lande, \& Schemske, 1984; Van der Niet, Peakall, \& Johnson, 2014; Weiblen, 2004). Yet, recent studies have begun to challenge the notion that interactions within this model system are tight, and growing evidence suggests that the posited one-to-one matching between figs and their pollinating wasps often breaks down (Cook \& Rasplus, 2003; Haine, Martin, \& Cook, 2006; Rasplus, 2011). How much these breakdowns repudiate current models of co-speciation (Hembry, Yoder, \& Goodman, 2014; Rasplus, 2011), and how much they might be an inherent part of the co-speciation process is currently unknown (Bronstein, Dieckmann, \& Ferrière, 2009). The resultant gaps and conflicts in current literature and theory demonstrate pressing need for a more complete and integrated knowledge of the mechanisms that govern co-speciation across the comprehensive continuum, from its incipient phases toward the full formation of new species.

While individual phases of co-speciation are well-documented in the literature (Cruaud et al., 2012; Weiblen, 2004), few studies have investigated co-speciation within a comparative framework to integrate previous results along the different stages of the speciation continuum. In the case of Yucca plants and their pollinating moths, it has been reported that congruence between the phylogenies of the interacting partners may result from the 
geographic tracking between the pollinators and their host plants, rather than from strict cospeciation (Althoff, Segraves, Smith, Leebens-Mack, \& Pellmyr, 2012; Hembry et al., 2014). In figs and fig wasps, wasp diversification is typically constrained by the host plants (Jousselin et al., 2008), which produces predominantly one-to-one patterns of co-speciation (Cruaud et al., 2012), despite the role that occasional duplications and host switches have also played in the mutualisms co-evolution (Cook \& Rasplus, 2003; Jousselin et al., 2008; Rasplus, 2011). Still, contrary to the expectation under strict co-speciation, where speciation events of interacting lineages occur at the same time (Huelsenbeck, Rannala, \& Larget, 2002), Moe \& Weiblen (2012) and Yu et al. (2019) have proposed faster speciation rates for wasps than their host figs, suggesting that occasional breakdowns in the one-to-one matching might be inevitable. While each of these studies provides useful insights, the results are hard to compare, as they span different regions, taxa, and different phases of the co-speciation process. To achieve a synthesis, we could benefit from a comparative approach that captures multiple phases of co-speciation within the same system.

\subsection{Co-speciation process and its underlying mechanisms}

Theoretical and empirical work suggests that the divergence patterns of one of the interacting partners could be associated with divergence from the other, progressing from increasing genetic divergence toward the complete formation of genetically well-defined species (Forister \& Feldman, 2011; Futuyma \& Agrawal, 2009). Specifically, pollinators have been hypothesized to promote reproductive isolation between plant populations during the initial phases of co-speciation (Hembry et al., 2014), which holds especially for pollinators with limited dispersal abilities that modulate gene flow between their host plant populations (Kiester et al., 1984; Souto-Vilarós et al., 2018; Van der Niet et al., 2014). These effects should be particularly evident in obligate nursery pollination systems, where pollinators are the sole means of pollen transfer between the host flowers. The pollinators, in turn, depend 
exclusively on the flowers that serve as oviposition site and food resource for their developing larvae (Althoff et al., 2012; Galil \& Eisikowitch, 1968; Kawakita, 2010). Growing genetic divergence within the host-plant populations should therefore be matched with the corresponding divergence across the populations of their pollinators (or vice versa), producing the characteristic pattern of co-speciation. Despite the extreme specificity of nursery pollinating systems, the one-to-one matching between host plants and their pollinators has been often reported to break down (Darwell, Al-Beidh, \& Cook, 2014; Li, Wang, \& Hu, 2015; Pellmyr, 1999; Yu et al., 2019). The causes of these breakdowns remain unclear. But one largely overlooked possibility is that the breakdowns represent an inherent part of the co-speciation process.

Cross-elevational comparisons may afford snapshots of genetic differentiation among the populations of figs and their pollinating wasps, representing different phases of the cospeciation process. Even though fig wasps are generally highly mobile dispersers, relying on passive dispersal by wind (Ahmed, Compton, Butlin, \& Gilmartin, 2009; Kobmoo, HossaertMckey, Rasplus, \& Kjellberg, 2010; Liu, Compton, Peng, Zhang, \& Chen, 2015), their mobility, and thereby the gene flow between their host plants, may sometimes be impaired. For instance, fig wasps associated with dioecious and under-canopy fig species have limited flight ability (Venkateswaran, Kumble, \& Borges, 2018), which is further compounded by the fact that dioecious fig trees tend to cluster into dense local populations (Dev, Kjellberg, Hossaert-Mckey, \& Borges, 2011; Wachi, Kusumi, Tzeng, \& Su, 2016). In contrast, wideranging monoecious species have little genetic structure over much of their ranges (Bain et al., 2016) and their pollinating wasps have been shown to be good dispersers (Venkateswaran et al., 2018). Additionally, differences in forest type, vegetation density, and dispersal capacity owing to wind, typical of different elevations within a montane system, likely produce elevational differences in gene flow. We expect varying levels of differentiation 
between the pollinator species from distinct evolutionary lineages (sections) of Ficus that colonized Mt Wilhelm during different stages of its orogeny. Consequently, each Ficus lineage likely represents a semi-independent radiation along the gradient. This makes crosselevation comparisons of population genetic structure between distinct lineages appealing for co-speciation research, as these may represent different stages along the speciation process.

\subsection{Gene flow and speciation along elevational gradients}

Elevational gradients have long been regarded as natural laboratories for the study of speciation (Körner, 2007; McCain \& Grytnes, 2010; Rahbek \& Museum, 1995). Tropical mountains in particular are often portrayed as 'speciation pumps', generating a disproportionate amount of the world's biodiversity (Kreft \& Jetz, 2007). The Andes of South America, Mount Cameroon in western Africa, Mount Kinabalu in Borneo and New Guinea's central range are among the most species-rich places on Earth and frequent subjects of macroevolutionary research (Rull, 2011; Toussaint et al., 2014). Restricted gene flow along the slopes of mountains, owing to environmental heterogeneity and non-random mating, likely promote population isolation, genetic differentiation, local adaptation and, eventually, parapatric speciation, explaining the high turnover of species with elevation (Byars, Parsons, Kirkpatrick \& Barton, 1997; Reis, Ciampi-Guillardi, Bajay, de Souza, \& dos Santos, 2015).

\subsection{Study system}

Here, we investigate the population genetic structure of six Ficus species (from four lineages, or sections) and their corresponding wasp pollinators along the Mount Wilhelm elevational gradient in New Guinea (NG). Fig species were selected to represent different stages of the co-speciation process: from continuous species with a wide elevational distribution, to parapatric, botanically recognized, sub-species, and finally, parapatric monophyletic species 
of their tight mutualism with pollinating fig wasps, our choice of host plant species therefore predetermined the choice of pollinating wasps, following the classic one-to-one relationship characteristic of this mutualism. We placed the fig species along the putative continuum according to existing phylogenetic, chemical (Souto-Vilarós et al., 2018), population genetic (Segar et al., 2017), and morphological information (Berg \& Corner, 2005). Further, the species were selected to be representative of an identifiable pattern of wide-ranging species (e.g. Ficus dammaropsis Diels), and recognized lowland and highland (sub)species pairs (e.g. F. saccata Corner/F. megalophylla Diels and F. trachypison K. Schum. /F. quercetorum Corner), in line with extensive field observations (Segar et al., 2017; L. Sam, unpublished data) and literature surveys (e.g. Berg and Corner, 2005). Our field collections started widely but focused in on species with high local abundance amenable to comparative analyses. While the selected fig species may not always be separated by known reproductive isolation barriers, the criteria detailed above indicate that they lie along different stages of the speciation continuum, which makes them suitable and of interest for population genetic comparisons. Such an approach, to date, is unique for this system and our study represents the only case where multiple fig and wasp species are studied together within a comparative framework.

\subsection{Hypothesized scenarios}

Within our study, we aim to identify genetic structure of populations of figs and their pollinating wasps in order to gauge the connectivity of individuals along their elevational ranges. Under the presumed one-to-one species specificity, we expect to find well connected population structure in both figs and wasps along their entire elevational distribution (Figure 1A). Alternatively, wasp local adaptation to the varying conditions along both extremes of the gradient, may promote more defined population structure. This structuring would in turn 
partners. Incomplete wasp specialization in the intermediate contact zone may be a mechanism by which genetic differentiation between highland and lowland fig subpopulations is minimized since even rare migrants can maintain gene flow between

177 populations (Figure 1B; Ellstrand, 2014). This mechanism can be further reinforced by 178 localized seed dispersal, which may additionally magnify the genetic structuring of fig populations.

Finally, further isolation between these parapatrically formed wasp clusters would continue to promote genetic distance between fig populations. Under this scenario, the accumulation of genetic dissimilarity is decoupled, such that parapatric wasp clusters (or incipient species) pollinate and thereby mediate the gene flow between fig subpopulations. This process eventually leads to the fig's speciation, which consequently restores of the one-to-one species matching between the figs and their wasps (Figure 1C).

For our study, we chose the New Guinean figs in the sub-genera Sycomorus and Sycidium.

This system is typified by very rare shifts of the pollinating wasps between their host plants (Moe, Rossi, \& Weiblen, 2011; Silvieus, Clement, \& Weiblen, 2008), such that co-speciation, rather than host shifts, typically explains the patterns of genetic matching between the plants and their pollinators. To rule out the possibility that host shifts might explain some of the detected patterns in population genetic structure, we built local phylogenies that included all con-generics sequenced to date. If host switching were common within this system, we would expect our focal species complexes to be non-monophyletic within this wider sampling context.

Even though our study system has the obvious advantage that associations between the interacting partners are clearly defined, given that one pollinator only enters one fig during its life time (female wasps often lose wings and antennae as they enter a fig, such that they cannot leave, and entering the 'wrong' species of fig consequently results in zero fitness), we 
cannot exclude the possibility that any limitation to genetic exchange between parapatric populations (as mediated by phenology and degrees of selfing, for example) could further influence the genetic structure of the studied populations. Nevertheless, our principal

202 objective here is not to definitively identify the causes of the detected genetic differentiation. Rather, we use the differentiation patterns to capture the co-speciation continuum, integrate the currently fragmentary results on co-speciation mechanisms, and evaluate previously formulated hypotheses on co-speciation along elevational gradients (Section 1.4), inspired by prominent literature in the field.

\section{Materials and Methods}

We chose six species-pairs of figs and their pollinating wasps and studied the genetic structure of their respective populations across elevations in the tropical montane system of New Guinea (200m - 2,700m; all elevations are given above sea level). DNA was extracted from 285 fig individuals and corresponding fig wasps and sequenced, using the NextRAD approach (Russello, Waterhouse, Etter, \& Johnson, 2015). This approach is suitable for samples with very low concentrations of DNA (as is typical of tiny fig wasps). The sequences were filtered to generate a dense SNP matrix for each species-pair, which we then processed through MCMC-based clustering algorithms to uncover the genetic structure of the examined populations. The uncovered clusters were compared against the predictions following from each of the three hypotheses on co-speciation dynamics (Figure 1). The comparisons consequently illuminated the mechanisms through which co-speciation operates within the fig-wasp system, at least within the examined tropical mountains.

\subsection{Study sites}

The present study was carried out at six sites along Mount Wilhelm in Papua New Guinea (PNG). All sites are spaced by approximately $500 \mathrm{~m}$ vertical increments, from 200 meters to 
2,700 metres, ranging from lowland alluvial forest up to lower montane forest. Site names and locations are described in Table 1. Forest types, broad species composition and general climatic conditions have been described in detail elsewhere (McAlpine, Keig, Falls, \& CSIRO, 1983; Sam, Koane, Jeppy, Sykorova, \& Novotny, 2017; Toussaint et al., 2014). We note that environmental conditions change rapidly along the transect, for example there is a $15^{\circ} \mathrm{C}$ temperature difference between our lowest and highest sites and a semi-permanent cloud layer at around 2,000 m.

\subsection{Focal Species}

Previous surveys of Ficus diversity carried out along the gradient (Segar et al., 2017; L. Sam, unpublished data) reported approximately 70 of the ca. 150 Ficus species present in PNG; however, pollinating fig-wasp species composition is unknown. Here, we studied five species and one taxonomically defined subspecies complex, all of which are endemic to New Guinea and adjacent islands (Berg \& Corner, 2005). We selected two, wide ranging, described species: F. wassa Roxb. (Sycidium) and F. arfakensis King (Sycocarpus), as representatives of species with wide elevational distributions. Their corresponding pollinating wasps are Kradibia wassae Wiebes and Ceratosolen solitarius Weibes, respectively, however, we identified multiple distinct wasp entities responsible for pollinating these fig species (see Results); F. trichocerasa Diels (Adenosperma) is a botanically recognized subspecies complex that replace each other in parapatry at approximately 1,700 m (Berg \& Corner, 2005); this complex represents an intermediate step along the speciation continuum where sub-species are not yet fully differentiated. Pollinating wasps of both subspecies are undescribed. Finally, we studied a monophyletic complex of three species (Souto-Vilarós et al., 2018) belonging to the section Papuacyse that follow a lowland, mid-elevation, and highland distribution, henceforth referred to as $F$. itoana species complex which includes: $F$. itoana Diels pollinated by Ceratosolen armipes Wiebes; F. microdictya Diels pollinated by 

wasp species-pair here referred to as Ficus sp. "IMI" and Ceratosolen sp. "IMI" (see also Souto-Vilarós et al. 2018). This complex represents a further step along the speciation continuum where each species-pair has differentiated enough to constitute a separate entity. Volatile organic compounds from both the Ficus itoana and F. trichocerasa complexes have been shown to differ between (sub)species, as well as pollinator preference for each of these emissions (Souto-Vilarós et al., 2018), which implicates co-evolution as the likely mechanism of cospeciation within these species (as opposed to, e.g., similar responses to geographic isolation, shared histories of immigration and colonization). Species names, breeding systems and distributions are detailed in Table 1, a map including collection sites and each individual tree sampled is presented in Figure 2.

We conducted sampling between August 2015 and November 2016. At each site we georeferenced and tagged between 10 and 15 individual trees of each locally available focal taxa. Nevertheless, in some instances, we were unable to find enough individuals for certain species at certain locations (i.e. F. itoana at the $200 \mathrm{~m}$ site). Similarly, due to differential rearing success of fig wasps, sampling size is in some cases imbalanced (see Table 1 for details). For each tree, we collected 10 to 15 leaf discs of $2.4 \mathrm{~cm}$ in diameter using a corkborer and stored them in colour changing silica gel for later DNA extraction and molecular analysis. In order to sustain wasp populations, fig trees fruit year round. Adult (and gravid) female wasps emerge from the trees in search of a fig in which to lay her eggs, however, fruiting is asynchronous within trees and so wasps regularly move away from their natal trees (Galil \& Eisikowitch, 1968). For each male tree tagged, we monitored fig development weekly or daily as necessary. Upon ripening to D-stage (the developmental stage when figwasps have hatched but not yet emerged from the fig; Galil \& Eisikowitch, 1968), we collected 10-15 figs and stored them in plastic pots covered with fine mesh to allow wasps to 
emerge. Once emerged, we collected ten female and ten male pollinating wasps for storage in $100 \%$ ethanol. A second collection of five males and five females was done using tubes filled with colour changing silica gel topped with cotton wool as per (Moe et al., 2011). For each tree, we used a single female fig-wasp from the silica gel collections for DNA extraction. We considered pooling of individuals inappropriate for such a fine-scale study and given that each fig typically contains many sisters (pollinating foundresses rarely leave a fig once inside and small figs are often pollinated by only one or two wasps) we maximised sampling above the fig level where possible. Finally, we collected and stored all remaining wasps in $100 \%$ ethanol. All samples were stored in a $-20^{\circ} \mathrm{C}$ freezer and shipped to the Czech Academy of Sciences, Czech Republic. Fig vouchers are stored in the National Herbarium in Lae, PNG, the New Guinea Binatang Research Centre (BRC), PNG, and the Czech Academy of Sciences, Czech Republic. Wasp vouchers are stored at the BRC and the Czech Academy of Sciences and Harper Adams University, UK.

\subsection{DNA extraction and nextRAD sequencing}

We performed DNA extractions from leaf discs using the CTAB protocol (Doyle \& Doyle, 1987) followed by an additional cleaning step using a silica column (Souto-Vilarós et al., 2018). From each individual fig, a single female pollinating wasp stored and dried in silica gel was used for DNA extraction. Isolations were performed using DNeasy Blood \& Tissue kits (Qiagen) following the manufacturers' protocol with the following modifications: Initial lysis was conducted at $37^{\circ} \mathrm{C}$ overnight followed by 30 minute incubation with $1 \mu \mathrm{L}$ of RNase (Qiagen) per sample. Final yield was further enhanced by using a total of $200 \mu \mathrm{L}$ of deionized water in two rounds of column washing. For both fig and wasp DNA samples, 1 $\mu \mathrm{L}$ of the final extract was used for QuBit quantification (QuBit 3 ThermoFisher Scientific) and $2 \mu \mathrm{L}$ were loaded on a GelRed® Nucleic Acid Gel Stain (Biotium) pre-stained 2\% agarose gel ran at $120 \mathrm{~V}$ for 70 minutes for visual inspection. Finally, fig DNA solutions 
were diluted to a total of $200 \mathrm{ng}$ in $40 \mu \mathrm{L}$ of EB buffer (Qiagen), while wasp DNA solutions (due to significantly lower yields) were vacuum dried and resuspended in $35 \mu \mathrm{L}$ of EB buffer to increase concentration to $20 \mathrm{ng}$ of DNA per sample.

Isolations were sent to SNPSAURUS, LLC for genotyping-by-sequencing (GBS) using Nextera-tagmented reductively amplified DNA sequencing (NextRAD) as per Russello et al., (2015). Genomic DNA was first fragmented with the Nextera reagent (Illumina, Inc), which also ligates short adapter sequences to the ends of the fragments. The Nextera reaction was scaled for fragmenting $5 \mathrm{ng}$ of genomic DNA for figs and $3 \mathrm{ng}$ for wasps, although $17.5 \mathrm{ng}$ and $6 \mathrm{ng}$ of genomic DNA for figs and wasps respectively was used as input to compensate for the amount of degraded DNA in the samples and to increase fragment sizes. For fig samples, fragmented DNA was then amplified for 26 cycles at $73^{\circ} \mathrm{C}$, with one of the primers matching the adapter and extending 9 nucleotides into the genomic DNA with the selective sequence GTGTAGAGC. For wasp samples, fragmented DNA was amplified for 25 cycles at $72^{\circ} \mathrm{C}$, with one of the primers matching the adapter and extending 8 nucleotides into the genomic DNA with the selective sequence GTGTAGAG. Thus, only fragments starting with a sequence that can be hybridized by the selective sequence of the primer was efficiently amplified. The nextRAD libraries were sequenced single end on a HiSeq 4000 with four lanes of $150 \mathrm{bp}$ reads (University of Oregon). Variant called format (VCF) files were generated by SNPSAURUS, LLC using custom scripts. First, reads from each sample were trimmed using the BBMAP tool bbduk (Bushnell, 2014). Trimming removed the Nextera adapter sequences and also quality trimmed the end of the reads as needed. Next, an equal number of reads were collected from each sample to create a catalogue of 10 million reads total. Read collection was done with BBTOOLS (BBMAP) with randomly selected reads. The catalogue was then analyzed to find sequences which may represent haplotypes in the population, by counting the instances of each sequence and 
keeping sequences with 10-1000 instances. These sequences were collapsed by aligning the sequences to each other with the BBToOLS program, using an alignment threshold of $85 \%$ identity. Reads that aligned to each other were considered different haplotypes of the same locus, and a single sequence of the aligning group was selected to represent that locus. Each sample's trimmed sequences were mapped to this de novo reference (using BBMAP), and the alignment piped to SAMTOOLS view to convert to a BAM file followed by a sorting step (using SAMTOOLS sort) to create a sorted BAM file for each sample (Heng Li et al., 2009). Genotype calling was done using mpileup (SAMTOOLS), using the de novo reference as a reference sequence and the list of sorted BAM files as input. This genotype table was further filtered using VCFTOOLS version 0.1.13 (Danecek et al., 2011) to remove alleles with low frequency in the population, which helps remove sequence error artefacts. The VCF file was also filtered by removing loci with all heterozygous genotypes or loci with more than 2 alleles at a single position, both of which suggest distinct loci had been collapsed together inappropriately. Specific commands for each step as well as additional dataset details (i.e. number of SNPs per species/individual) are provided as supplementary material, VCF files for each species used in our analysis are available from the Dryad Digital Repositori (doi:10.5061/dryad.5m4dn73). Raw demultiplexed NextRAD sequences are available from the Short Read Archive (NCBI) BioProject number PRJNA555181.

We generated molecular barcodes for 86 wasps (representative of each identified genomic cluster; see Results) using cytochrome oxidase $b$ (cyt-b), a marker with high specificity and amplification rates in fig wasps which has the ability to delimit closely related fig wasp species (Lopez-Vaamonde et al., 2009; Segar, Lopez-Vaamonde, Rasplus, \& Cook, 2012). Due to unsuccessful amplification of cyt- $b$ for individuals of Ceratosolen sp. "IMI," we generated COI sequences of 4 individuals to compare them against previously published sequences for wasps of the $F$. itoana complex. PCR primers and conditions for both 
fragments are outlined in Cruaud et al. (2010). Similarly, in order to establish the role of host shifting in our system we tested the monophyly of wasp clades (see Results) through the reconstruction of wider molecular phylogenies using the F2 copy of elongation factor 1alpha, (ef1-a) and cyt- $b$. We amplified ef1-a using the primers reported by Cruaud et al. (2011) and concatenated both markers for a total of 92 individuals (50 Kradibia and 42

Ceratosolen) spread across the genetic clusters identified. We provide a breakdown of data collected for each individual wasp (nextRAD-seq, cyt- $b$ and ef1-a) in the supplementary material, when all DNA was used for NextRAD sequencing of alternative individuals were made.

\subsection{Data analysis}

Population genetic structure was inferred using FINERADSTRUCTURE (Malinsky, Trucchi, Lawson, \& Falush, 2018), a pipeline specifically designed for the analysis of RAD data using the FINESTRUCTURE Markov chain Monte Carlo (MCMC) clustering algorithm (Lawson, Hellenthal, Myers, \& Falush, 2012). Without previous assumptions of populations, the program generates a "co-ancestry matrix" between all individuals and collection sites which is then combined with MCMC sampling to identify clusters of individuals which share the highest degree of estimated relatedness. We converted VCF files to the required format using custom python scripts (supplementary material). The software RADPAINTER, as implemented in the FINERADSTRUCTURE package, was then used to calculate the co-ancestry matrix. Individuals were assigned to populations using 100,000 MCMC replications and a burn-in of 10,000 as per default settings (Malinsky et al., 2018). Tree building was conducted using the default parameters and results were visualized using $\mathrm{R}$ scripts (available at http://cichlid.gurdon.cam.ac.uk/fineRADstructure.html). Because our data is not sorted according to genome coordinates, linkage disequilibrium (LD) between RAD loci is rather 
conservative number of identifiable population clusters, prior to calculating the co-ancestry matrix, RAD loci were reordered according to LD using the sampleLD.R script implemented in the package (Malinsky et al., 2018). An initial analysis was done including all samples to generate a co-ancestry matrix for all figs (Figure S1; supplementary material), and fig-wasps (Figure S2; supplementary material) in order to identify the relative relatedness of all samples. Further analyses were done per species in order to increase resolution of the identified groups.

Levels of genetic differentiation between collection sites were estimated by pairwise $\mathrm{F}_{\mathrm{ST}}$ from the VCF files using the Weir and Cockerham (Weir \& Cockerham, 1984) method as implemented in VCFTOOLS version 0.1.13 (Danecek et al., 2011).

We used the $c y t-b$ barcodes to obtain molecular operational taxonomic units (MOTUs) for wasps and used jMOTU v.1.0.7 (Jones, Ghoorah, \& Blaxter, 2011) and the web version of ABGD (Automatic Barcode Gap Discovery for primary species delimitation; Puillandre, Lambert, Brouillet, \& Achaz, 2012) in order to distinguish whether or not retrieved wasp required to address reproductive isolation between them and that ongoing gene flow (e.g. mtDNA introgression) may prevent complete resolution in many cases (Whitworth, Dawson, Magalon, \& Baudry, 2007). Nevertheless, this method provides an objective way of defining provide more robust delimitation. jMOTU derives genetic distances from pair-wise Needleman-Wunsch alignments, selecting representatives of each resultant cluster for allagainst-all comparisons. The number of clusters (MOTUs) is reported for a given set of 
barcode gap, after which increasing genetic dissimilarity results in a stable number of

400

401

402

403

404

405

406

407

408

409

410

411

412

413

414

415

416

417

418

419

420

421

422

clusters. The barcode gap lower limit represents the genetic distance just beyond the upper limits of intra-specific variation. ABGD derives a threshold by using a range of genetic similarities to determine the extent of intra-specific variation and cluster MOTUs, subsequent recursive partitioning is used to partition that data and determine the best estimate of the barcode gap as well as the corresponding number of MOTUs. Using jMOTU we set the low BLAST identity filter to 97 but otherwise settings were left as default. For ABGD we set the minimum genetic distance to 0.001 and the maximum to 0.4 . The number of steps was set to 200 and the relative gap width was set to 0.75 . The number of bins was set to 40 . We used the Kimura K80 distance with the TS/TV ratio set to the default 2.0. The barcode gap was identified using jMOTU by plotting the number of MOTUs vs. the percentage cut-off and finding the value over which the MOTU numbers plateau following a sharp decrease which results from intra-specific clustering (the results of both analyses are presented as supplementary material). This was compared to the number of MOTUs found from the barcode gap as estimated by ABGD, the barcode gap is well represented by the histogram of genetic distances between sequences: the barcode gap is the region between intra-specific and inter-specific distances (Puillandre et al., 2012).

Phylogenetic analyses were performed separately for Ceratosolen and Kradibia wasps and alignments of both cyt- $b$ and ef1-a markers were concatenated. We included only the individuals for which we had sequences for ef1-a, and complemented them with cyt- $b$ sequences. In addition to the generated sequences, we included published sequences for pollinating fig wasps which may co-occur at our study site obtained by Cruaud et al. (2012). Alignment was conducted using MUSCLE (Edgar, 2004) and tree building using maximum likelihood (ML) as implemented in RAxML-HPC BlackBox (Stamatakis, 2014) using $\mathrm{HKY}+\mathrm{G}$ and $\mathrm{HKY}+\mathrm{I}+\mathrm{G}$ substitution models for each species (Ceratosolen and Kradibia 
respectively) selected according to Bayesian information criterion as per JMODELTEST 2 version 0.1.10 (Darriba, Taboada, Doallo, \& Posada, 2012). The final alignments for

Ceratosolen included 76 individuals while the Kradibia alignment included 67 sequences.

427

Sequences from the genus Dolichoris were used as outgroup in both instances.

\section{Results}

Co-ancestry heat maps generated using FINERADSTRUCTURE provide detailed population structure for both figs and fig wasps. For F. wassa and F. arfakensis, we were able to detect highland and lowland populations of figs, often with mid-elevation contact zones. Low pairwise fixation index $\left(\mathrm{F}_{\mathrm{ST}}\right)$ values suggest these to be well connected populations indicating that some of these species do have wide elevational distributions. Contrastingly, our analysis on fig-wasps reveal more tightly clustered entities along the gradient with very high $\mathrm{F}_{\mathrm{ST}}$ values suggesting infrequent exchange of genes; supplementary genetic barcoding analyses show inter-specific distances in mitochondrial DNA commensurate with species level splits, however, whether or not these entities are reproductively isolated from each other remains unknown. For the two widely distributed fig species, our analysis suggests multiple highly differentiated genetic entities along the gradient while for the subspecies and sister species complex, it is likely that individual wasp species are responsible for pollinating each of the fig (sub)species maintaining the classic one-to-one species specificity. Importantly, within our study, we place emphasis on the comparative approach that draws on multiple species, while exercising caution when interpreting the results of any one comparison on its own, especially when sample sizes are low.

3.1 Ficus wassa: two populations of figs with a contact zone and more than three clusters of wasps 
Figure 3 illustrates the level of co-ancestry between $F$. wassa (Figure 3A) as well as its Kradibia pollinators (Figure 3B). Ficus wassa has a wide elevational distribution from sea level to 2,700 m (Berg \& Corner, 2005). At our sites we find highland and lowland population clusters, while the mid-elevations (here defined as 1,700 m) appear to represent a contact zone. $\mathrm{F}_{\mathrm{ST}}$ values (Figure $5 \mathrm{~A}$ ), however, reveal little genetic differentiation between sites ( $\mathrm{F}_{\mathrm{ST}}$ between sites $<0.01$ ), suggesting these clusters to be populations of a wide-ranging species. Given that $F$. wassa has been traditionally cultivated by humans, it seems possible that this pattern may have been, at least partly, influenced by human-mediated dispersal. Pollinating wasps display a contrasting pattern with a very closely related and sub-structured lowland cluster composed by most wasps from the $200 \mathrm{~m}$ site and a second group from the 700 and 1,200 $\mathrm{m}$ sites. There is further evidence of a deep split between lowland and highland pollinator clusters. $\mathrm{F}_{\mathrm{ST}}$ values between wasp populations are correspondingly high (Figure $5 \mathrm{~B}$ ) while $\mathrm{F}_{\mathrm{ST}}$ values within the clusters show moderate genetic diversity (700 m vs. $1,200 \mathrm{~m} \mathrm{~F}_{\mathrm{ST}}<0.09$; between highland populations $\left.\mathrm{F}_{\mathrm{ST}}<0.14\right)$. According to our mtDNA analyses, these three clusters are certainly distinct enough to constitute separate MOTUs (with pairwise distance above $2.5 \%$ likely representing the barcode gap according to jMOTU; Figure S3). Nevertheless, the co-ancestry analysis (Figure 3B) shows one individual from the $200 \mathrm{~m}$ site grouping with the $700 \mathrm{~m}$ and 1,200 m cluster, and one 1,200 m individual with the highland group. This may be due to incomplete wasp specialization and consequently maintaining a certain level of gene flow between fig populations, particularly at the midelevation contact zone. However, the exact interpretation remains uncertain due to the small sample sizes involved. Finally, mtDNA suggests even deeper splits and an extra fourth (an additional highland cluster), or even fifth MOTU (an additional lowland cluster, according to ABGD). Although the barcoding gap is ambiguous within this clade perhaps reflecting recent ancestry and incomplete lineage sorting or mtDNA introgression (Figure S3). 


\subsection{Ficus arfakensis: three populations of figs and four clusters of wasps}

474 Equivalent plots are given for $F$. arfakensis (Figure 3C) and its corresponding pollinator(s)

475

476

477

478

479

480

481

482

483

484

485

486

487

488

489

490

491

492

493

494

495

496

497

(Figure 3D). These plots suggest a split between highland $(1,700 \mathrm{~m})$ and lowland fig populations (below $700 \mathrm{~m}$ ) and a distinct third entity at mid-elevations $(1,200 \mathrm{~m})$. The situation seems comparable to that of $F$. wassa as $\mathrm{F}_{\mathrm{ST}}$ values are moderate $\left(\mathrm{F}_{\mathrm{ST}}<0.15\right.$ for all comparisons; Figure 5C) suggesting that $F$. arfakensis occupies a broad elevational distribution with a low degree of gene-flow between otherwise distinct populations. The wasp data shows four clear groups: two sympatric wasp clusters occurring in the lowlands (between 200 and $700 \mathrm{~m}$ ), a third cluster at 1,200 $\mathrm{m}$ and a final cluster at 1,700 $\mathrm{m}$. $\mathrm{F}_{\mathrm{ST}}$ values between these wasp clusters retrieve high genetic diversity for each comparison (Figure 5D) and jMOTU analysis retrieved four distinct MOTUs (a result corroborated by ABGD), with a distinct barcoding gap at $2.5 \%$ (Figure S3). As in the case above, wasps typical of the midelevation site (1,200 m) are found at lower elevations (Figure 3D), suggesting that incomplete pollinator specialisation occurs.

\subsection{Ficus trichocerasa: two subspecies of figs and three clusters of wasps}

Figure 4 shows the co-ancestry matrix for $F$. trichocerasa subsp. trichocerasa and $F$. trichocerasa subsp. pleioclada (Figure 4A) and their corresponding fig-wasps (Figure 3B). Ficus trichocerasa has two clear and genetically defined clusters which correspond to the described ranges of both subspecies (Berg \& Corner, 2005; Souto-Vilarós et al., 2018). F ST $_{\text {T }}$ values between subspecies show high genetic dissimilarity $\left(\mathrm{F}_{\mathrm{ST}}>0.24\right)$ while there appears to be very little dissimilarity within subspecies at different sites $\left(\mathrm{F}_{\mathrm{ST}}<0.07\right.$; Figure $\left.5 \mathrm{C}\right)$. Similarly, wasp individuals cluster according to the populations of their host subspecies. Unfortunately, due to a combination of limited rearing success and tree availability at the $1,200 \mathrm{~m}$ site, we were only able to retrieve information from three individual wasps which appear to constitute a third cluster or contact zone between subspecies, which makes any 
clear generalizations for this species unwarranted. However, $\mathrm{F}_{\mathrm{ST}}$ values suggest these to be closely related to the $700 \mathrm{~m}$ population $\left(\mathrm{F}_{\mathrm{ST}}=0.07\right)$ while they appear to be quite distinct from the highland $(1,700-2,200 \mathrm{~m})$ population $\left(\mathrm{F}_{\mathrm{ST}}>0.80\right.$; Figure $\left.5 \mathrm{C}\right)$. Our barcoding results from both approaches supported this finding of three wasp clusters (Figure S3). We successfully barcoded four individuals of Ceratosolen associated with $F$. trichocerasa at $1,200 \mathrm{~m}$ with three of these individuals clustering alongside all individuals from $700 \mathrm{~m}$, and one forming a singleton cluster at a barcode threshold of $1 \%$; thus, while more samples at this site would further clarify the relationship between wasps at this contact zone, there is a clear distinction between lowland and highland pollinators (Figure S3).

\subsection{Ficus itoana complex: three species of figs and three species of wasps}

508 Finally, Figure 4 shows the co-ancestry matrix for all species in the $F$. itoana complex $(F$. itoana, F. sp. "IMI" and F. microdictya; Figure 4C) and their corresponding fig-wasps (Ceratosolen armipes, C. sp. "IMI" and C. "kaironkensis"; Figure 4D). Our analyses recover three distinct clusters corresponding to each of the three species. According to the coancestry heat map, Ficus sp. "IMI" appears to be closely related to its sister species $F$. microdictya $\left(\mathrm{F}_{\mathrm{ST}}=0.16\right)$, however, these two species have distinct breeding systems and pollinating fig-wasps (Souto-Vilarós et al., 2018). Interesting to note is that one $F$. sp. "IMI" individual appears to have shared ancestry between $F$. microdictya and $F$. sp. "IMI" suggesting it to be a hybrid between both. Pairwise $\mathrm{F}_{\mathrm{ST}}$ comparisons between species clusters show moderate to high genetic differentiation $\left(\mathrm{F}_{\mathrm{ST}}\right.$ between 0.16 and 0.34 ; Figure 5D). In the case of the pollinating fig-wasps of this complex, we identify three clear clusters corresponding to each of the host species and $\mathrm{F}_{\mathrm{ST}}$ values between comparisons show high genetic differentiation between pollinating fig-wasps $\left(\mathrm{F}_{\mathrm{ST}}>0.63\right.$; Figure 5D). We were unable to amplify cyt- $b$ sequences for all but one pollinating wasp of $F$. sp. "IMI," however, 522 the morphological and behavioural differences described by Souto-Vilarós et al. (2018) 
provide compelling evidence that this is indeed a separate species. Similarly, a BLAST

524 (Madden, 2003) search for highly similar sequences (megablast) on the NCBI nucleotide

525

526

527 collection database of our COI sequences for these individuals found that these sequences are equally dissimilar to those from $C$. armipes and $C$. "kaironkensis", which are the closest matches. This final comparison is the only one where incomplete pollinator specialisation was not found (but see Souto-Vilarós et al., 2018).

\subsection{Molecular phylogenies}

We recovered well resolved molecular phylogenies (Figure S4) that were in broad agreement with those in published studies (e.g. Cruaud et al., 2012), albeit using fewer taxa and markers. The main result was that the monophyly of our molecular clusters was maintained, with no evidence for host shifting in this system. Furthermore, we were able to recover the major RAD-seq derived clusters, adding further support for their validity and demonstrating that the distances between described species are commensurate with those between our genetic clusters.

\section{Discussion}

Our findings reveal a co-speciation continuum that spans six species of figs and their pollinating wasps within the tropical mountains of New Guinea. The genetic structure of the examined populations is consistent with a scenario where wasps diverge faster than their host figs, generating locally adapted clusters along the elevation gradient during the early stages of co-speciation. The newly formed clusters of wasps then likely restrict pollen movement, which contributes to the observed genetic structure of the fig populations. Restricted pollen movement, potentially further compounded by restricted seed dispersal, may lead to reproductive isolation between the fig populations leading to their eventual speciation. Under this scenario, one-to-one matching breaks down during the intermediate phases of co- 
speciation when the same species of a fig may be pollinated by multiple localized species of wasps. The matching is later restored, as the wasp clusters impose novel reproductive barriers to gene flow among fig populations culminating in their speciation. These findings uncover the mechanisms of co-speciation in a hallmark study system, which could be informative of the broader dynamics operating among plants and their pollinators.

553 While other explanations for the detected patterns in our genetic data are possible, our interpretation is consistent with classic work on mathematical modelling which suggests that any small pool of figs, which differ enough to restrict gene flow, could co-evolve with their pollinating wasps and thereby promote co-speciation (Kiester et al., 1984). Further, we found that wasps experience far greater limitations to gene flow along the gradient than do figs, as evidenced by the tight clustering of wasp populations, higher $\mathrm{F}_{\mathrm{ST}}$ values between populations than within them, and confirmed by DNA barcoding and phylogenetic analyses. These wasp clusters may in turn promote character divergence within fig ecotypes (for example through volatile preference). Importantly, our findings held across multiple pairs of interacting species and across different stages of the speciation process. Still, further work is required both in terms of taxa and regions studied. In particular, future studies across the central range of New Guinea would illuminate if the same differentiation emerges in parallel along different mountain slopes. Recent work has reported nine species of pollinating wasps, distributed parapatrically within the geographic range of their host, Ficus hirta Vahl (Yu et al., 2019), suggesting that different wasp species might be pollinating the same fig species at different locations, even when every location shows only one pollinator. Should the cospeciation dynamics we found operate more broadly across different montane systems, there may be far more pollinating fig-wasps per host fig than typically presumed.

571 Our results integrate and extend previously reported findings on co-speciation. In line with 572 the accumulating evidence against the one-to-one species specificity in the fig and fig wasp 
mutualism (Cook \& Rasplus, 2003; Rasplus, 2011), we find that the one-to-one matching occasionally breaks down. We also identify the circumstances when the breakdowns occur, and illustrate that they represent an inevitable intermediate phase of the co-speciation process (Fig. 3B, D). Such process of "split and sort speciation" has been previously proposed in the literature (Cook \& Segar, 2010). Our results indicate that the breakdowns may not be exceptions to the rule, but rather a transitional, and therefore only rarely reported, phase of co-speciation. Further, we find that speciation rates in host plants may be decoupled from speciation rates of their pollinators, which likely holds in other systems as well (e.g. Yuccas and Yucca moths), especially when the interacting partners have dramatically different lifecycles, generation times, and genetic divergence rates (Moe, Clement, \& Weiblen, 2012). Finally, our results connect the previous evidence for different phases of speciation into one process (Nosil, Harmon, \& Seehausen, 2009) and thereby provide mechanistic insights about the progression of co-speciation, from its incipient phases toward the complete formation of new species (Figure 1). Further research into the mechanisms, however, remains warranted. In particular, a definitive proof of co-speciation resulting from co-evolution (e.g. rather than from similar responses to geographic isolation) would require demonstrating a match between the signal genes in figs (scent pathways) and receiver genes in wasps (olfactory receptors) (Segar, Volf, Sisol, Pardikes, \& Souto-Vilarós, 2019). While the genomic data for such analyses are becoming increasingly available (Mori et al., 2017; Xiao et al., 2013) they have yet to be compiled for our study system. Genomic matching of the corresponding genes in plants and pollinators stands out as the necessary next step toward the mechanistic understanding of co-speciation patterns (Segar et al., 2019).

\subsection{Caveats and alternative explanations}

Our results may have been influenced by multiple classes of confounding factors. It is possible that the genetic differentiation is influenced by co-occurring species of figs and their 
pollinating wasps. For example, host-switching could represent an alternative explanation for some of the detected patterns in our genomic data. While we cannot exclude such effects completely, our phylogenetic results dispute that such effects could be prominent, given that we found strong support for the monophyly of the retrieved wasp clusters. Co-speciation, rather than host switching, therefore seems to acts as a plausible explanation for the genetic patterns detected. This interpretation corresponds with much of the previous work that has shown that switching, although important in the evolution of current associations, is rare where the survival and reproduction of both interacting partners are coupled (Jousselin et al., 2008). In line with our phylogenetic results, evidence from extensive studies including $F$. arfakensis, $F$. wassa and twenty additional sympatric fig species both within a phylogenetic (Silvieus et al., 2008) and at the population level (Moe et al., 2012) recover congruent fig and wasp relationships and only limited evidence for host switching within this system. Admittedly, a comprehensive phylogeny including all sympatric figs and wasps is the only definitive way to rule out host switching. Still, we placed our wasp species into the most densely sampled phylogeny available to date. We would therefore expect to find at least some evidence for host shifts if these played relevant role in our system. Yet, we found none. In addition to host shifts, immigration followed by colonization could also explain the genetic patterns observed. However, our fig species have been reported throughout the entire island of New Guinea (Berg \& Corner, 2005) and genetic analyses confirmed that the species are not dispersal limited (Segar et al., 2017). This indicates that adaptation (e.g. to climate at different elevations), rather than dispersal, limits the geographic distribution of figs and their co-speciation with fig wasps.

Further, while our sample sizes were limited, especially for some species, they were sufficient to recover robust support for some of the evaluated hypotheses (Figure 1) and identify the speciation continuum across the wasps and their host figs in a comparative 
context. Nevertheless, there are a few instances where additional wasp samples would

624

625

626

627

628

629

630

631

632

633

634

635

636

637

638

639

640

641

642

643

644

645

646

647 certainly strengthen our conclusions. Specifically, more wasp samples from the $F$. trichocerasa complex at the $1,200 \mathrm{~m}$ site would help clarify whether or not this constitutes a third cluster, as retrieved by jMOTU analysis, or if it represents a hybrid zone between pollinators of both subspecies (as revealed by the ancestry analysis). Similarly, increasing wasp sample size for the highland $F$. wassa wasps could help refine the structuring of these populations. We further acknowledge that our results cannot definitively establish the presence of reproductive isolation barriers among the studied wasp clusters. Still, phylogenetic and mtDNA results tentatively indicate that the wasp clusters were divergent enough to constitute separate species. Moreover, we argue that the relevance of our results lies in comparing different phases of the co-speciation process, and such comparisons are interesting in their own right, regardless of whether the process has produced reproductive isolation. Finally, we note that low rearing success in mid- and high elevations had biological foundations, including low pollination rates in mid-elevation $F$. itoana (potentially a sign of hybrid depression) and exceptional (non-wasp) parasite loads at the higher range edge of $F$. wassa (2,700m) as has been reported also for other fig species (Piatscheck, Van Goor, Houston, \& Nason, 2018).

\subsection{Mechanisms of co-speciation in figs and their pollinating wasps}

Within montane systems, environmental conditions change dramatically even over short distances, which typically imposes steep reproductive barriers in both plants and animals (Bachman, Baker, Brummitt, Dransfield, \& Moat, 2004; Körner, 2007; Reis et al., 2015; Rieseberg \& Willis, 2007). Figs and fig pollinating wasps are known to have co-evolved over millions of years (Cruaud et al., 2012; Jousselin et al., 2008), and still, it has been proposed that speciation dynamics in nursery pollination mutualisms may sometimes be decoupled (Hembry \& Althoff, 2016). Currently, it is uncertain whether speciation events are due to 
strict co-speciation, host shifts, and/or duplications (Cook \& Segar, 2010). It appears that when ecological gradients are less pronounced, plants do not diversify while their pollinating wasps might. For example, Ficus hirta appears to form a cline across its large continental and oceanic range and in this case, a recent study found up to nine pollinating wasp species along its range (Yu et al., 2019). Contrastingly, Kobmoo et al. (2010) found that a single population of Ceratosolen fusciceps Mayr pollinates F. racemosa L. throughout most of South-East Asia, a relatively homogeneous, but large biogeographic region. Similarly, the authors also found a genetically distinct wasp entity in mainland Australia pollinating the same fig species suggesting two pollinating species. Likewise, studies on Pleistodontes imperialis Saunders (the pollinator of $F$. rubiginosa Desf. ex. Vent.) found limited gene flow and possible cryptic wasp species over a wide latitudinal gradient in eastern Australia (Darwell et al., 2014). Our results reveal a similar pattern, albeit at a far smaller scale, with structured fig populations across an elevational gradient, and multiple pollinating wasps for species with broad elevational distributions. It appears that environmental heterogeneity and discrete geographic barriers play an important role in the local isolation of fig populations giving rise to rapid isolation of pollinating wasps resulting in the formation of new species, without the necessity of a new host fig. We suggest that both temperature gradients and cloud cover will pose problems to dispersing fig wasps, indeed the largest genetic turnover is found between 1,200 $\mathrm{m}$ and 2,200 m. Horizontal distances are generally small between our study sites $(<10 \mathrm{~km})$ and isolation by distance is not found in F. arfakensis across larger distances ( 80km; Segar et al., 2017). Due to their short life-span, assortative mating within the fig before emergence, and weak dispersal abilities, specifically for below-canopy pollinators (Dev et al., 2011; Wachi et al., 2016), fig wasps seem to speciate along their host's range, perhaps aided by a resistance to Allee effects and genetic incompatibilities driven by Wolbachia (Yu et al., 2019). This structuring within wasps may eventually restrict pollen movement, promoting fig 
673 population structure and ultimately, host fig speciation (Cook \& Segar, 2010). Competitive 674 exclusion of less well adapted fig wasps (Liu et al., 2014) might also restore one-to-one 675 pollination similar to sympatric non-pollinating fig wasps diverging in ovipositor length, and 676 likely niche (Darwell \& Cook, 2017). Of more immediate significance is the fact that 677 pollination within evolving species groups is not always entirely specific, and our results suggest that mid-elevation pollinator individuals can move one site up or down the transect. This renders the exact reason for connectivity between populations of figs open for debate, and suggests that species sorting of wasps may occur before ancestral fig species fully split.

\subsection{Insights from the speciation continuum}

We base our conclusions on the comparisons across the species examined. Such comparative approach holds the promise of revealing generalities while being less sensitive to the possible differences in species' genetic makeup, dispersal history, and the employed sample size. Our data shows that for broadly ranging fig species (i.e. F. wassa and F. arfakensis), populations at different elevations host different species of wasps. In both cases, pollinator specificity and dispersal abilities may restrict gene flow between fig populations while a certain degree of connectivity between them maintains species integrity, possibly through seed dispersal. Speciation may be at a particularly early stage in $F$. wassa, with the exchange of wasps and genes between populations a distinct possibility. It has been reported that immigrant seed gene flow may have evolutionary significant consequences over wide distances, maintaining otherwise isolated entities as a single species (Ellstrand, 2014). Historical cultivation of $F$. wassa could also have contributed to the increased connectivity between the populations. However, we specifically avoided abandoned gardens and restricted our sampling to undisturbed forests to mitigate the effects of human-mediated dispersal. continuum, where divergent populations have developed distinct volatile profiles (Souto- 
Vilarós et al., 2018) and morphological differences (which become less clear at the contact zone, between 1,200 - 1,500 m ; Berg \& Corner, 2005). The development of such differences suggests these taxa are in the early stages of ecological speciation, even though they are not

701

702

703

704

705

706

707

708

709

710

711

712

713

714

715 yet totally differentiated (Nosil et al., 2009; Wu, 2001). Since scent is believed to be a major trait in pollinator attraction in the fig-wasp mutualism (Hossaert-McKey et al., 2016; Proffit et al., 2009), ecological isolation due to pollinator preference for each subspecies may eventually lead to the formation of fully separate fig species. The $F$. itoana complex represents an advanced stage in the speciation process where all three species are pollinated by a corresponding wasp species and all three pairs are separated parapatrically following a lowland (F. itoana), mid-elevation ( $F$. sp. "IMI") and highland (F. microdictya) distribution. In this case, the formation of new fig species potentially restored the classic one-to-one species specificity of such specialized mutualisms.

\subsection{Restricted dispersal of both pollen and seeds as promoters of plant speciation}

Besides pollen movement, in our system restricted to pollinating fig wasps, a key mechanism maintaining plant genetic connectivity is the movement of seeds across the landscape. Our focal species have a variety of fruit syndromes (Table 1) which suggest different seed dispersing guilds (Lomáscolo, Levey, Kimball, Bolker, \& Alborn, 2010; Shanahan, 2000; Shanahan, So, Compton, \& Corlett, 2001). Seed dispersal by birds may have a different effect depending on the bird species. Previous bird surveys at the Mt. Wilhelm elevational gradient have demonstrated that bird communities change with elevation and only a few species are found throughout the entire gradient (Marki et al., 2016; Sam \& Koane, 2014; Sam et al., 2017). Similarly, fruit bats have been shown to disperse fig seeds (Shilton, Altringham, Compton, \& Whittaker, 1999; Thornton et al., 2001), but, the range over which they disperse differs according to species. Bat abundance decreases sharply with elevation at our transect (P. Amick, unpublished data) suggesting that dispersal by bats may be restricted to lower 
elevations, while dispersal by small understory animals, such as bandicoots (M.S. pers. obs.) is restricted to their home ranges. Studies in Prunus mahaleb L. (Rosaceae) found different relative contributions of seed dispersers to genetic connectivity in the studied population between open and closed microhabitats. Small birds were shown to be responsible for shortdistance dispersal while larger birds and mammals account for long-distance dispersal demonstrating that different frugivores contribute differently to seed-mediated gene flow (Jordano, Garcia, Godoy, \& Garcia-Castano, 2007). It has been suggested that limited seed dispersal in turn limits pollen dispersal due to higher local tree density further isolating already diverging populations (Hardy et al., 2006). We propose that the next step to better understand the drivers of fig population structure and speciation could be to couple our results with collecting information on seed dispersers and the distances at which they are able to disperse seeds.

\subsection{Barriers to gene flow in continuous populations}

Speciation can unfold across environmental gradients, even in the absence of discrete barriers. Gene flow along a gradient is often mediated by the interplay of biotic and abiotic factors, which may eventually lead to speciation. For instance, continuous populations of Anthoxantum odoratum L. (Poaceae) growing along a pollution gradient across a heavy metal mine boundary experience limitations to gene flow, particularly at the contact zone, through differences in flowering time (Antonovics, 2006). These phenological differences promote assortative mating between metal tolerant and non-tolerant plants, effectively maintaining genetic divergence (Antonovics, 2006). Animal pollination can further reinforce these barriers promoting local adaptation and avoid swamping of maladapted genes (Kirkpatrick \& Barton, 1997). This can be seen in the insect pollinated alpine grass Poa hiemata Vickery (Poaceae) in which flowering time differs between highland and lowland populations, leading to reduced opportunities for pollinators, causing genetic structuring between elevations 
748 (Byars et al., 2009). Even in plants pollinated by a wide range of insects, genetic structuring between lowland and highland species has been described and attributed, in part, to effective pollinator availability at different elevations (Reis et al., 2015). Feedbacks, whereby insects

751 become adapted to local conditions and limit the flow of maladapted genotypes between plant individuals, may result in even stronger barriers to gene flow, and enhance the rates of diversification (Armbruster \& Muchhala, 2009). Specialised pollination mutualisms offer an even more tractable system to measure the influence of biotic and abiotic mechanisms promoting plant divergence. In the case of Yucca brevifolia, the two varieties studied by Godsoe et. al. (2009) at different geographic distributions appear to be the result of coevolution with their corresponding moth pollinators rather than abiotic conditions (Godsoe et al., 2009). Our results for figs and their pollinating wasps extend this growing spectrum of examples. They also demonstrate how biotic interactions along the continuum of environmental conditions (temperature, productivity, changing vegetation types, etc.) can result in speciation.

\subsection{Conclusion}

Figs and wasps present a remarkable system with to study co-speciation, from the early phases of genetic differentiation to the establishment of gene flow barriers. Our results demonstrate that mutualistic interactions, often implicated in speciation bursts and the generation of new diversity (Schemske, Mittelbach, Cornell, Sobel, \& Roy, 2009), tend to break down occasionally. We also demonstrate the evolutionary consequences of the breakdowns in mutualistic interactions, and reveal how they reciprocally foster co-speciation across the interacting partners. Knowing which of the previously reported breakdowns represent an intermediate step in co-speciation and which an adverse side effect of other perturbations (e.g. human-induced changes in the biotic milieu) may be relevant for conservation. Further, we find that speciation tends to be imbalanced, even within the 
constraints of a tight mutualism. Specifically, pollinators, owing to their faster life-cycles,

774

775

776

777

778

779

780

781

782

783

784

785

786

787

788

789

790

791

792

793

794

795

796

shorter generation times, and faster genetic differentiation, may speciate significantly faster than their host plants. Together, these findings demonstrate how new diversity of figs and their pollinating wasps is generated within an understudied system famous for its extraordinary diversity, the mountain slopes of tropical New Guinea. They also pave the way toward a more integrated and nuanced understanding of the speciation process involving multiple interacting partners and, consequently, illuminate the mechanisms behind the interplay of species interactions and environmental gradients, whose confluence presumably contributes significantly to the accumulation of species diversity.

\section{Acknowledgements}

We thank all our field assistants at each of the collection sites as well as camp managers and all staff at the New Guinea Binatang Research Centre. We thank the Papua New Guinea Forest Research Institute and the Department of Environment and Conservation for help in getting plant and insect export permits. We thank the National Grid and Infrastructure MetaCentrum for computing and storage facilities provided under the programme "Projects of Large Research, Development and Innovations Infrastructures” (CESNET LM2015042). Thanks to the Workshop of Speciation and Population Genomics (2018) for in depth discussions and examples on the analysis of NGS data. We thank Finn Kjellberg, four anonymous reviewers and the handling editor for their constructive review towards the improvement of this manuscript. A.M. was supported by the European Union's Horizon 2020 research and innovation programme under the Marie Sklodowska-Curie grant agreement No. 785799 and by Charles University Research Centre program No. 204069. S.T.S. acknowledges funding from the Grant Agency of the Czech Republic (number 15-24511S) 
797 and departmental support from Harper Adams University. V.N. acknowledges ERC 669609

798 funding.

799 


\section{References}

Ahmed, S., Compton, S. G., Butlin, R. K., \& Gilmartin, P. M. (2009). Wind-borne insects mediate directional pollen transfer between desert fig trees 160 kilometers apart. Proceedings of the National Academy of Sciences, 106, 20342-20347. doi:10.1073/pnas.0902213106

Althoff, D. M., Segraves, K. A., Smith, C. I., Leebens-Mack, J., \& Pellmyr, O. (2012). Geographic isolation trumps coevolution as a driver of yucca and yucca moth diversification. Molecular Phylogenetics and Evolution, 62, 898-906. doi:10.1016/j.ympev.2011.11.024

Antonovics, J. (2006). Evolution in closely adjacent plant populations X: Long-term persistence of prereproductive isolation at a mine boundary. Heredity, 97, 33-37. doi:10.1038/sj.hdy.6800835

Armbruster, W. S., \& Muchhala, N. (2009). Associations between floral specialization and species diversity: Cause, effect, or correlation? Evolutionary Ecology, 23, 159-179. doi:10.1007/s10682-008-9259-Z

Bachman, S., Baker, W. J., Brummitt, N., Dransfield, J., \& Moat, J. (2004). Elevational gradients, area and tropical island diversity: An example from the palms of New Guinea. Ecography, 27, 299-310. doi:10.1111/j.0906-7590.2004.03759.x

Bain, A., Borges, R. M., Chevallier, M. H., Vignes, H., Kobmoo, N., Peng, Y. Q., ... Hossaert-Mckey, M. (2016). Geographic structuring into vicariant species-pairs in a wide-ranging, high-dispersal plant-insect mutualism: the case of Ficus racemosa and its pollinating wasps. Evolutionary Ecology, 30, 663-684. doi:10.1007/s10682-016-9836-5

Berg, C. C., \& Corner, E. J. H. (2005). Flora Malesiana, Series I. Volume 17 part 2. In Flora Malesiana Series I - Seed Plants vol. 17 Part 2 (pp. 1-730).

Bronstein, J. L., Dieckmann, U., \& Ferrière, R. (2009). Coevolutionary Dynamics and the Conservation of Mutualisms. In R. Ferrière, U. Dieckmann, \& D. Couvet (Eds.), Evolutionary Conservation Biology (pp. 305-326). Cambridge: Cambridge University Press. doi:10.1017/cbo9780511542022.022

Bushnell, B. (2014). BBMap: a fast, accurate, splice-aware aligner. Joint Genome Institute, department of energy. doi:/10.1186/1471-2105-13-238

Byars, S. G., Parsons, Y., \& Hoffmann, A. A. (2009). Effect of altitude on the genetic structure of an Alpine grass, Poa hiemata. Annals of Botany, 103, 885-899. doi:10.1093/aob/mcp018

Caro, L. M., Caycedo-Rosales, P. C., Bowie, R. C. K., Slabbekoorn, H., \& Cadena, C. D. (2013). Ecological speciation along an elevational gradient in a tropical passerine bird? Journal of Evolutionary Biology, 26, 357-374. doi:10.1111/jeb.12055

Cook, J. M., \& Rasplus, J. Y. (2003). Mutualists with attitude: Coevolving fig wasps and figs. Trends in Ecology and Evolution. doi:10.1016/S0169-5347(03)00062-4

Cook, J. M., \& Segar, S. T. (2010). Speciation in fig wasps. Ecological Entomology, 35, 5466. doi:10.1111/j.1365-2311.2009.01148.x

Cruaud, A., Jabbour-Zahab, R., Genson, G., Cruaud, C., Couloux, A., Kjellberg, F., ... Rasplus, J. Y. (2010). Laying the foundations for a new classification of Agaonidae (Hymenoptera: Chalcidoidea), a multilocus phylogenetic approach. Cladistics, 26, 359387. doi:10.1111/j.1096-0031.2009.00291.x

Cruaud, A., Ronsted, N., Chantarasuwan, B., Chou, L. S., Clement, W. L., Couloux, A., ... Savolainen, V. (2012). An Extreme case of plant-insect codiversification: Figs and figpollinating wasps. Systematic Biology, 61, 1029-1047. doi:10.1093/sysbio/sys068

Danecek, P., Auton, A., Abecasis, G., Albers, C. A., Banks, E., DePristo, M. A., ... Durbin, R. (2011). The variant call format and VCFtools. Bioinformatics, 27, 2156-2158. doi:10.1093/bioinformatics/btr330 
Darriba, D., Taboada, G. L., Doallo, R., \& Posada, D. (2012). JModelTest 2: More models, new heuristics and parallel computing. Nature Methods. doi:10.1038/nmeth.2109

Darwell, C. T., \& Cook, J. M. (2017). Cryptic diversity in a fig wasp communitymorphologically differentiated species are sympatric but cryptic species exhibit competitive exclusion. Molecular Ecology, 26, 937-950. doi:10.1111/mec.13985

Darwell, Clive T., Al-Beidh, S., \& Cook, J. M. (2014). Molecular species delimitation of a symbiotic fig-pollinating wasp species complex reveals extreme deviation from reciprocal partner specificity. BMC Evolutionary Biology, 14, 189. doi:10.1186/s12862014-0189-9

Dev, S. A., Kjellberg, F., Hossaert-Mckey, M., \& Borges, R. M. (2011). Fine-Scale Population Genetic Structure of Two Dioecious Indian Keystone Species, Ficus hispida and Ficus exasperata (Moraceae). Biotropica, 43, 309-316. doi:10.1111/j.17447429.2010.00704.x

Doyle, J. J., \& Doyle, J. L. (1987). A rapid DNA isolation procedure for small quantities of fresh leaf tissue. Phytochemical Bulletin, 19, 11-15. doi:10.2307/4119796

Edgar, R. C. (2004). MUSCLE: multiple sequence alignment with high accuracy and high throughput. Nucleic Acids Research. doi:10.1093/nar/gkh340

Ehrlich, P. R., \& Raven, P. H. (2006). Butterflies and Plants: A Study in Coevolution. Evolution, 18, 586-608. doi:10.2307/2406212

Ellstrand, N. C. (2014). Is gene flow the most important evolutionary force in plants? American Journal of Botany, 101, 737-753. doi:10.3732/ajb.1400024

Forister, M. L., \& Feldman, C. R. (2011). Phylogenetic cascades and the origins of tropical diversity. Biotropica, 43, 270-278. doi:10.1111/j.1744-7429.2010.00702.x

Futuyma, D. J., \& Agrawal, A. A. (2009). Macroevolution and the biological diversity of plants and herbivores. Proceedings of the National Academy of Sciences, 106, 1805418061. doi:10.1073/pnas.0904106106

Galil, J., \& Eisikowitch, D. (1968). Flowering Cycles and Fruit Types of Ficus sycomorus in Israel. New Phytologist, 67, 745-758. doi:10.1111/j.1469-8137.1968.tb05497.x

Godsoe, W., Strand, E., Smith, C. I., Yoder, J. B., Esque, T. C., \& Pellmyr, O. (2009). Divergence in an obligate mutualism is not explained by divergent climatic factors. New Phytologist. doi:10.1111/j.1469-8137.2009.02942.x

Haine, E. R., Martin, J., \& Cook, J. M. (2006). Deep mtDNA divergences indicate cryptic species in a fig-pollinating wasp. BMC Evolutionary Biology, 6, 1-11. doi: 10.1186/1471-2148-6-83

Hardy, O. J., Maggia, L., Bandou, E., Breyne, P., Caron, H., Chevallier, M. H., ... Degen, B. (2006). Fine-scale genetic structure and gene dispersal inferences in 10 Neotropical tree species. Molecular Ecology, 15, 559-571. doi:10.1111/j.1365-294X.2005.02785.x

Hembry, D. H., \& Althoff, D. M. (2016). Diversification and coevolution in brood pollination mutualisms: Windows into the role of biotic interactions in generating biological diversity. American Journal of Botany. doi:10.3732/ajb.1600056

Hembry, D. H., Yoder, J. B., \& Goodman, K. R. (2014). Coevolution and the Diversification of Life. The American Naturalist, 184, 425-438. doi:10.1086/677928

Hossaert-McKey, M., Proffit, M., Soler, C. C. L., Chen, C., Bessière, J.-M., Schatz, B., \& Borges, R. M. (2016). How to be a dioecious fig: Chemical mimicry between sexes matters only when both sexes flower synchronously. Scientific Reports, 6, 21236. doi:10.1038/srep21236

Huelsenbeck, J. P., Rannala, B., \& Larget, B. (2002). A statistical perspective for reconstructing the history of host-parasite associations. In R. D. M. Page (Ed.), Tangled Trees: Phylogenies, Cospeciation and Coevolution (pp. 93-119). Chicago: Chicago University Press. 
Jones, M., Ghoorah, A., \& Blaxter, M. (2011). JMOTU and taxonerator: Turning DNA barcode sequences into annotated operational taxonomic units. PLOS ONE, 6. doi:10.1371/journal.pone.0019259

Jordano, P., Garcia, C., Godoy, J. A., \& Garcia-Castano, J. L. (2007). Differential contribution of frugivores to complex seed dispersal patterns. Proceedings of the National Academy of Sciences, 104, 3278-3282. doi:10.1073/pnas.0606793104

Jousselin, E., Van Noort, S., Berry, V., Rasplus, J.-Y., Rønsted, N., Erasmus, J. C., \& Greeff, J. M. (2008). One fig to bind them all: Host conservatism in a fig wasp community unraveled by cospeciation analyses among pollinating and nonpollinating fig wasps. Evolution, 62, 1777-1797. doi:10.1111/j.1558-5646.2008.00406.x

Kawakita, A. (2010). Evolution of obligate pollination mutualism in the tribe Phyllantheae (Phyllanthaceae). Plant Species Biology, 25, 3-19. doi:10.1111/j.14421984.2009.00266.x

Kiester, A. R., Lande, R., \& Schemske, D. W. (1984). Models of Coevolution and Speciation in Plants and Their Pollinators. The American Naturalist, 124, 220-243. doi: $10.1086 / 284265$

Kirkpatrick, M., \& Barton, N. H. (1997). Evolution of a species' range. The American Naturalist, 150, 1-23. doi:10.1086/286054

Kobmoo, N., Hossaert-Mckey, M., Rasplus, J.-Y., \& Kjellberg, F. (2010). Ficus racemosa is pollinated by a single population of a single agaonid wasp species in continental SouthEast Asia. Molecular Ecology, 19, 2700-2712. doi:10.1111/j.1365-294X.2010.04654.x

Körner, C. (2007). The use of "altitude" in ecological research. Trends in Ecology and Evolution, 22, 569-574. doi:10.1016/j.tree.2007.09.006

Kreft, H., \& Jetz, W. (2007). Global patterns and determinants of vascular plant diversity. Proceedings of the National Academy of Sciences, 104, 5925-5930. doi:10.1073/pnas.0608361104

Lawson, D. J., Hellenthal, G., Myers, S., \& Falush, D. (2012). Inference of population structure using dense haplotype data. PLoS Genetics, 8. doi:10.1371/journal.pgen.1002453

Li, Heng, Handsaker, B., Wysoker, A., Fennell, T., Ruan, J., Homer, N., ... Durbin, R. (2009). The Sequence Alignment/Map format and SAMtools. Bioinformatics, 25, 20782079. doi:10.1093/bioinformatics/btp352

Li, Houhun, Wang, Z., \& Hu, B. (2015). Four new species of Epicephala Meyrick, 1880 (Lepidoptera, Gracillariidae) associated with two species of Glochidion (Phyllanthaceae) from Hainan Island in China. ZooKeys, 508, 53-67. doi:10.3897/zookeys.508.9479

Liu, M., Compton, S. G., Peng, F. E., Zhang, J., \& Chen, X. Y. (2015). Movements of genes between populations: Are pollinators more effective at transferring their own or plant genetic markers? Proceedings of the Royal Society B: Biological Sciences, 282, 1-9. doi:10.1098/rspb.2015.0290

Liu, M., Zhao, R., Chen, Y., Zhang, J., Compton, S. G., \& Chen, X. Y. (2014). Competitive exclusion among fig wasps achieved via entrainment of host plant flowering phenology. PLoS ONE, 9, e97783. doi:10.1371/journal.pone.0097783

Lomáscolo, S. B., Levey, D. J., Kimball, R. T., Bolker, B. M., \& Alborn, H. T. (2010). Dispersers shape fruit diversity in Ficus (Moraceae). Proceedings of the National Academy of Sciences of the United States of America, 107, 14668-14672. doi:10.1073/pnas.1008773107

Lopez-Vaamonde, C., Wikström, N., Kjer, K. M., Weiblen, G. D., Rasplus, J. Y., Machado, C. A., \& Cook, J. M. (2009). Molecular dating and biogeography of fig-pollinating wasps. Molecular Phylogenetics and Evolution, 52, 715-726. doi:10.1016/j.ympev.2009.05.028 
Madden, T. (2003). The BLAST Sequence Analysis Tool. In The NCBI Handbook[internet]. doi:10.1086/597785

Malinsky, M., Trucchi, E., Lawson, D. J., \& Falush, D. (2018). RADpainter and fineRADstructure: Population Inference from RADseq Data. Molecular Biology and Evolution, 35, 1284-1290. doi:10.1093/molbev/msy023

Marki, P. Z., Sam, K., Koane, B., Bolding Kristensen, J., Kennedy, J. D., \& Jønsson, K. A. (2016). New and noteworthy bird records from the Mt . Wilhelm elevational gradient, Papua New Guinea Study area and Methods. Bulletin of the British Ornithologists 'Club, 136, 263-271.

McAlpine, J. R., Keig, G., Falls, R., \& CSIRO. (1983). Climate of Papua New Guinea. Canberra: Commonwealth Scientific and Industrial Research Organization in association with Australian National University Press.

McCain, C. M., \& Grytnes, J.-A. (2010). Elevational Gradients in Species Richness. Encylcopedia of Life Sciences, 15, 1-10. doi:10.1002/9780470015902.a0022548

Moe, A. M., Clement, W., \& Weiblen, G. D. (2012). Rapid evolution of pollinator-mediated plant reproductive isolation. In R. Singh, X. Jianping, \& R. Kulathinal (Eds.), Rapidly Evolving Genes and Genetic Systems (pp. 266-273). Oxford University Press.

Moe, A. M., Rossi, D. R., \& Weiblen, G. D. (2011). Pollinator sharing in dioecious figs (Ficus: Moraceae). Biological Journal of the Linnean Society, 103, 546-558. doi:10.1111/j.1095-8312.2011.01669.x

Moe, A. M., \& Weiblen, G. D. (2012). Pollinator-mediated reproductive isolation among dioecious fig species (Ficus, moraceae). Evolution, 66, 3710-3721. doi:10.1111/j.15585646.2012.01727.x

Mori, K., Shirasawa, K., Nogata, H., Hirata, C., Tashiro, K., Habu, T., ... Ikegami, H. (2017). Identification of RAN1 orthologue associated with sex determination through whole genome sequencing analysis in fig (Ficus carica L.). Scientific Reports, 7, 41124. doi:10.1038/srep41124

Nosil, P., Harmon, L. J., \& Seehausen, O. (2009). Ecological explanations for (incomplete) speciation. Trends in Ecology and Evolution. doi:10.1016/j.tree.2008.10.011

Pellmyr, O. (1999). Systematic revision of the yucca moths in the Tegeticula yuccasella complex (Lepidoptera: Prodoxidae) north of Mexico. Systematic Entomology, 24, 243271. doi:10.1046/j.1365-3113.1999.00079.x

Piatscheck, F., Van Goor, J., Houston, D. D., \& Nason, J. D. (2018). Ecological factors associated with pre-dispersal predation of fig seeds and wasps by fig-specialist lepidopteran larvae. Acta Oecologica. doi:10.1016/j.actao.2018.03.001

Proffit, M., Chen, C., Soler, C., Bessière, J. M., Schatz, B., \& Hossaert-Mckey, M. (2009). Can chemical signals, responsible for mutualistic partner encounter, promote the specific exploitation of nursery pollination mutualisms? - The case of figs and fig wasps. Entomologia Experimentalis et Applicata, 131, 46-57. doi:10.1111/j.15707458.2009.00823.x

Puillandre, N., Lambert, A., Brouillet, S., \& Achaz, G. (2012). ABGD, Automatic Barcode Gap Discovery for primary species delimitation. Molecular Ecology, 21, 1864-1877. doi:10.1111/j.1365-294X.2011.05239.x

Rahbek, C., \& Museum, Z. (1995). The elevational gradient of species richness: a uniform pattern? Ecography, 2, 200-205. doi:10.1111/j.1600-0587.1995.tb00341.x

Rasplus, J.-Y. (2011). The one-to-one species specificity of the Ficus-Agaoninae mutualism: how casual? In L. G. J. van der Maesen, X. M. van der Burgt, \& J. M. van der Medenbach de Rooy (Eds.), The Biodiversity of African Plants (pp. 639-649). Dordrecht: Kluwer Academic Publishers. doi:10.1007/978-94-009-0285-5_78

Reis, T. S., Ciampi-Guillardi, M., Bajay, M. M., de Souza, A. P., \& dos Santos, F. A. M. 
(2015). Elevation as a barrier: Genetic structure for an Atlantic rain forest tree (Bathysa australis) in the Serra do Mar mountain range, SE Brazil. Ecology and Evolution, 5, 1919-1931. doi:10.1002/ece3.1501

Rieseberg, L. H., \& Willis, J. H. (2007). Plant Speciation. Science, 317, 910-914.

Rull, V. (2011). Neotropical biodiversity: Timing and potential drivers. Trends in Ecology and Evolution, 26, 508-513. doi:10.1016/j.tree.2011.05.011

Russello, M. A., Waterhouse, M. D., Etter, P. D., \& Johnson, E. A. (2015). From promise to practice: pairing non-invasive sampling with genomics in conservation. PeerJ, 3, e1106. doi:10.7717/peerj.1106

Sam, K., \& Koane, B. (2014). New avian records along the elevational gradient of Mt. Wilhelm, Papua New Guinea. Bulletin of the British Ornithologists' Club, 134, 116133.

Sam, K., Koane, B., Jeppy, S., Sykorova, J., \& Novotny, V. (2017). Diet of land birds along an elevational gradient in Papua New Guinea. Scientific Reports, 7, 44018. doi:10.1038/srep44018

Schemske, D. W., Mittelbach, G. G., Cornell, H. V., Sobel, J. M., \& Roy, K. (2009). Is There a Latitudinal Gradient in the Importance of Biotic Interactions? Annual Review of Ecology, Evolution, and Systematics, 40, 245-269. doi:10.1146/annurev.ecolsys.39.110707.173430

Segar, S.T., Volf, M., Sisol, M., Pardikes, N. A., \& Souto-Vilarós, D. (2019). Chemical cues and genetic divergence in insects on plants: conceptual cross pollination between mutualistic and antagonistic systems. Current Opinion in Insect Science, 32, 83-90. doi:10.1016/j.cois.2018.11.009

Segar, S. T., Volf, M., Zima, J., Isua, B., Sisol, M., Sam, L., ... Novotny, V. (2017). Speciation in a keystone plant genus is driven by elevation: a case study in New Guinean Ficus. Journal of Evolutionary Biology, 30, 512-523. doi:10.1111/jeb.13020

Segar, S. T., Lopez-Vaamonde, C., Rasplus, J. Y., \& Cook, J. M. (2012). The global phylogeny of the subfamily Sycoryctinae (Pteromalidae): Parasites of an obligate mutualism. Molecular Phylogenetics and Evolution, 65, 116-125. doi:10.1016/j.ympev.2012.05.030

Shanahan, M. (2000). Ficus seed dispersal guilds: ecology, evolution and conservation implications. Ph.D. thesis. University of Leeds, UK.

Shanahan, M., So, S., Compton, S. G., \& Corlett, R. T. (2001). Fig-eating by vertebrate frugivores: a global review. Biological Reviews, 76, 529-572. doi:10.1017/S1464793101005760

Shilton, L. A., Altringham, J. D., Compton, S. G., \& Whittaker, R. J. (1999). Old World fruit bats can be long-distance seed dispersers through extended retention of viable seeds in the gut. Proceedings of the Royal Society B: Biological Sciences, 266, 219-223. doi:10.1098/rspb.1999.0625

Silvieus, S. I., Clement, W. L., \& Weiblen, G. D. (2008). Cophylogeny of Figs, Pollinators, Gallers, and Parasitoids. In K. Tilmon (Ed.), Specialization, speciation and radiation: The evolutionary biology of herbivorous insects (First, pp. 225-239). Berkley and Los Angeles: University of California Press.

Souto-Vilarós, D., Machac, A., Michalek, J., Darwell, C. T., Sisol, M., Kuyaiva, T., ... Segar, S. T. (2019). Data from: Faster speciation of fig-wasps than their host figs leads to decoupled speciation dynamics: snapshots across the speciation continuum. Dryad Digital Repository. doi:10.5061/dryad.5m4dn73

Souto-Vilarós, D., Proffit, M., Buatois, B., Rindos, M., Sisol, M., Kuyaiva, T., ... Segar, S. T. (2018). Pollination along an elevational gradient mediated both by floral scent and pollinator compatibility in the fig and fig-wasp mutualism. Journal of Ecology, 106, 

2256-2273. doi:10.1111/1365-2745.12995

Stamatakis, A. (2014). RAxML version 8: A tool for phylogenetic analysis and post-analysis of large phylogenies. Bioinformatics, 30, 1312-1313. doi:10.1093/bioinformatics/btu033

Thornton, I. W. B., Cook, S., Edwards, J. S., Harrison, R. D., Schipper, C., Shanahan, M., ... Yamuna, R. (2001). Colonization of volcano long Islands Papua New Guinea and an emergent island Motmot in its caldera lake Overview and discusion. Journal of Biogeography, 28, 1389-1408. doi:10.1046/j.1365-2699.2001.00638.x

Toussaint, E. F. A., Hall, R., Monaghan, M. T., Sagata, K., Ibalim, S., Shaverdo, H. V., ... Balke, M. (2014). The towering orogeny of New Guinea as a trigger for arthropod megadiversity. Nature Communications, 5, 4001. doi:10.1038/ncomms5001

Van der Niet, T., Peakall, R., \& Johnson, S. D. (2014). Pollinator-driven ecological speciation in plants: New evidence and future perspectives. Annals of Botany, 113, 199211. doi:10.1093/aob/mct290

Venkateswaran, V., Kumble, A. L. K., \& Borges, R. M. (2018). Resource dispersion influences dispersal evolution of highly insulated insect communities. Biology Letters, 14. doi:10.1098/rsbl.2018.0111

Wachi, N., Kusumi, J., Tzeng, H. Y., \& Su, Z. H. (2016). Genome-wide sequence data suggest the possibility of pollinator sharing by host shift in dioecious figs (Moraceae, Ficus). Molecular Ecology, 25, 5732-5746. doi:10.1111/mec.13876

Weiblen, G. D. (2004). Correlated evolution in fig pollination. Systematic Biology, 53, 128139. doi:10.1080/10635150490265012

Weir, B. S., \& Cockerham, C. C. (1984). Estimating F-Statistics for the Analysis of Population Structure. Evolution, 38, 1358-1370.

Whitworth, T. L., Dawson, R. D., Magalon, H., \& Baudry, E. (2007). DNA barcoding cannot reliably identify species of the blowfly genus Protocalliphora (Diptera: Calliphoridae). Proceedings of the Royal Society B: Biological Sciences, 274, 1731 - 1739. doi:10.1098/rspb.2007.0062

$\mathrm{Wu}, \mathrm{C}$. I. (2001). The genic view of the process of speciation. Journal of Evolutionary Biology, 14, 851-865. doi:10.1046/j.1420-9101.2001.00335.x

Xiao, J.-H., Yue, Z., Jia, L.-Y., Yang, X.-H., Niu, L.-H., Wang, Z., ... Huang, D.-W. (2013). Obligate mutualism within a host drives the extreme specialization of a fig wasp genome. Genome Biology, 14, R141. doi:10.1186/gb-2013-14-12-r141

Yu, H., Tian, E., Zheng, L., Deng, X., Cheng, Y., Chen, L., ... Kjellberg, F. (2019). Multiple parapatric pollinators have radiated across a continental fig tree displaying clinal genetic variation. Molecular Ecology. doi:10.1111/mec.15046 
1088 Data used for this study are available from the Dryad Digital Repository http:// 10.5061/dryad.5m4dn73 (Souto-Vilarós et al., 2019). Cytocrome - b, Elongation factor 1090 alpha and COI sequences are deposited in GenBank. Accession numbers MN168894 1091 BMN169018 (broken down per individual in supplementary material). Demultiplexed 1092 NextRAD sequence data is available from the Short Read Archive (NCBI) BioProject ID 1093 PRJNA555181.

1094

\section{Author's contributions}

1095

V.N., S.T.S. and D.S.V. planned the research with guidance of G.D.W. and B.I. for suitable 1096 focal species. S.T.S., D.S.V., M.S., B.I. and T.K. conducted the fieldwork and managed all 1097 field assistants while not on site. D.S.V. and J.M. conducted and managed all aspects of the 1098 molecular laboratory while C.T.D. assisted with the NGS data management and analysis.

1099 D.S.V., A.M. and S.T.S. analysed the data and wrote the manuscript with substantial input 1100 from all authors. All authors contributed and approved the final version of the manuscript. 
Table 1. Collection sites, focal Ficus species and corresponding wasps collected from each location. Numbers within parenthesis for pollinators indicate which population cluster they belong to as revealed by FINERADSTRUCTURE analysis. Dispersal syndrome is derived from fruit morphology

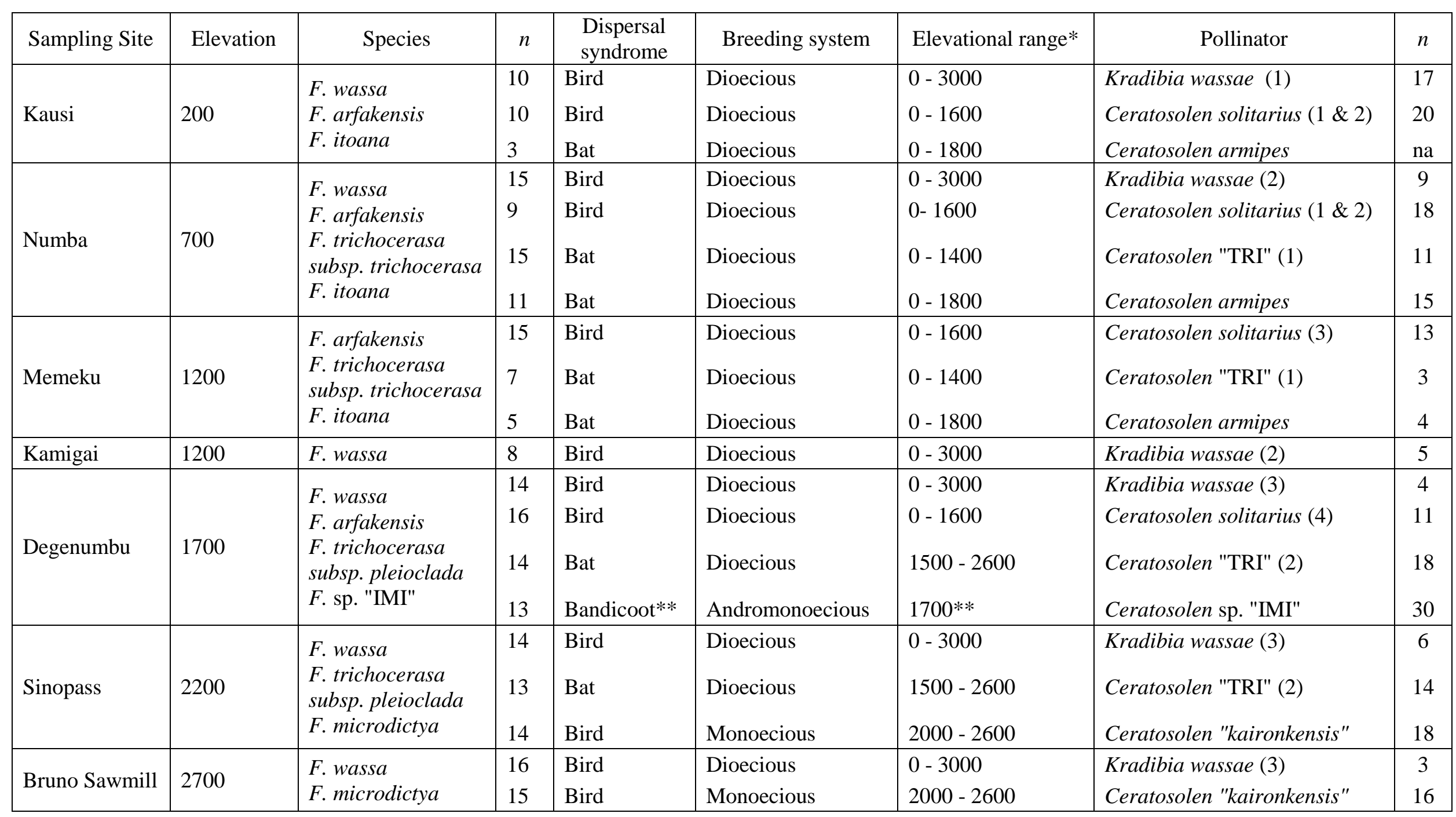

* Species distribution taken from Berg \& Corner 2005

** personal observation 


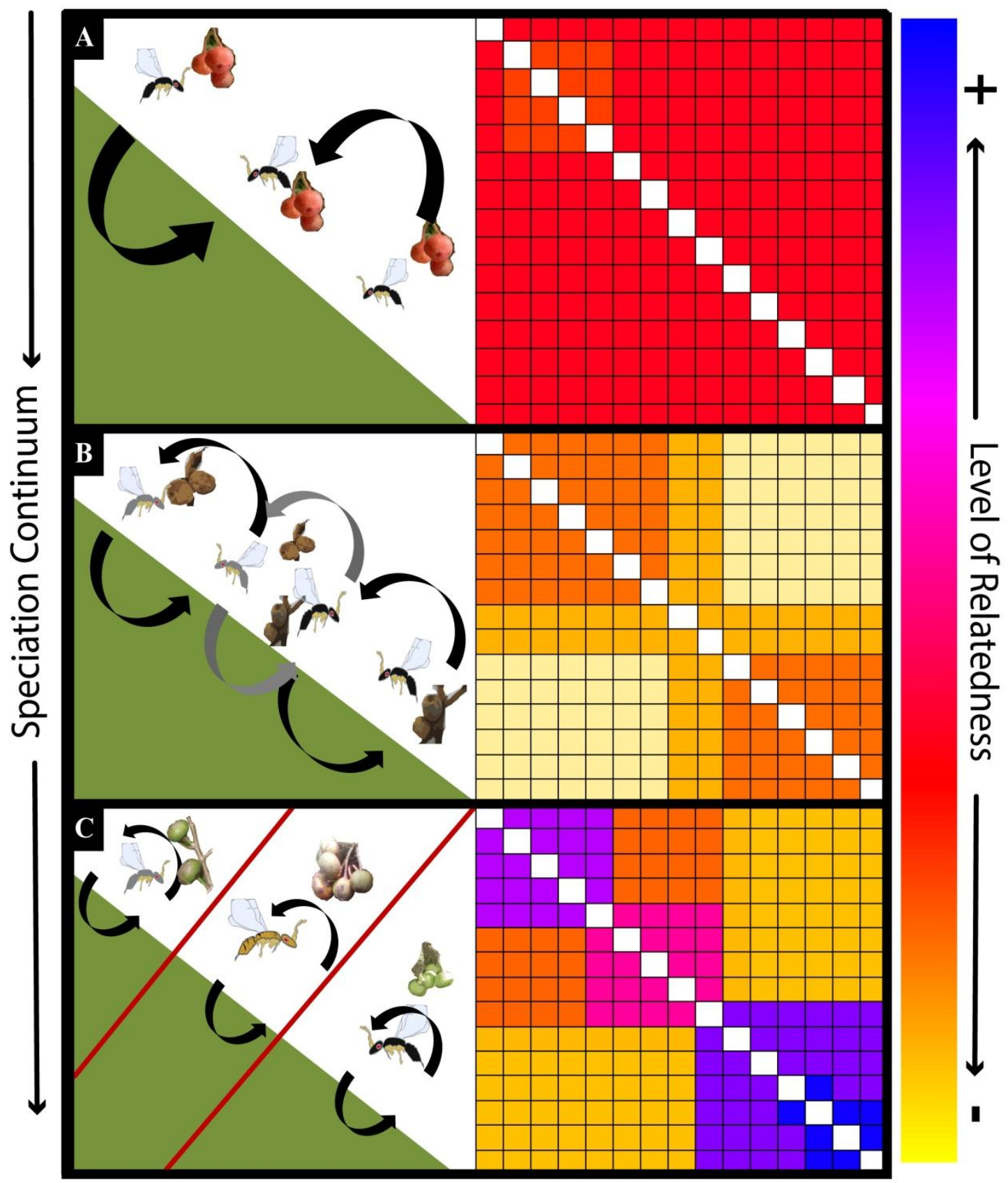

Figure 1. Three stages of the co-speciation process and the genetic patterns expected under each of them. Fig wasps in the classic one-to-one scenario (A) maintain genetic connectivity and population structure of their fig host across its distribution along an elevational gradient. Fig wasps at both extremes of the gradient adapt locally (B) and thus, influence host fig population structure; some degree of occasional gene flow at the contact zone due to incomplete fig wasp specialization, maintains limited host fig connectivity. Increasing reproductive isolation between fig populations lead to their speciation $(\mathrm{C})$, restoring the oneto-one scenario for parapatrically distributed fig species. Heat maps mimic the co-ancestry matrix, darker colours indicate higher level of relatedness among populations. Black arrows indicate pollen flow, gray arrows indicate occasional pollen flow between fig ecotypes or subspecies; red lines represent barriers to geneflow. 


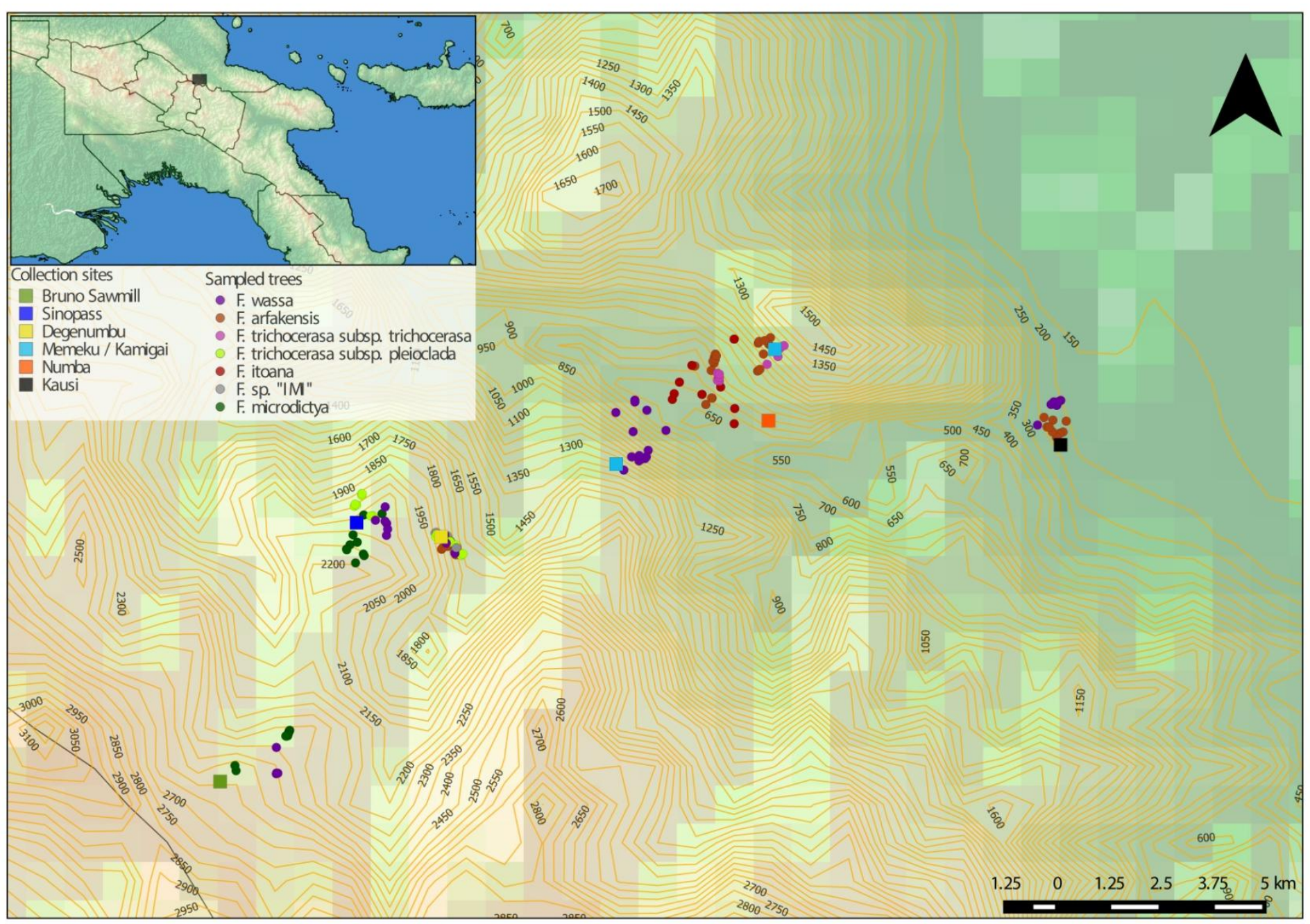

1117

Figure 2. Geographic location of field sites (squares) and sampled trees (circles) along the Mount Wilhelm elevational gradient in Madang province, Papua New Guinea (note that there are two sites at the 1,200 m elevation due to a lack of $F$. wassa individuals at the original Memeku 1120 site) 


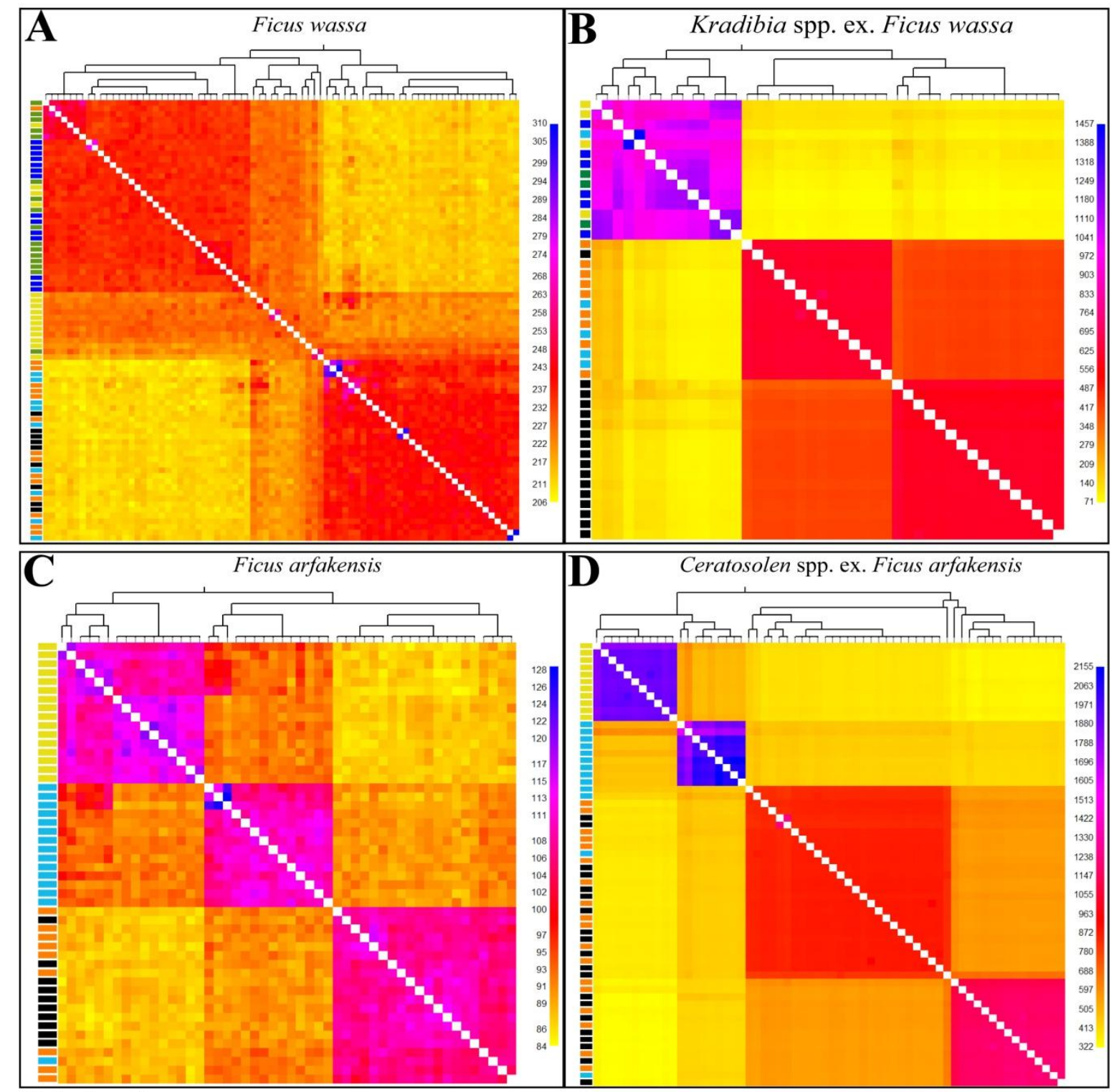

\section{Collection sites}

Kausi - 200 masl

Numba - 700 masl

Memeku -1,200 masl

Degenumbu - 1,700 masl

Sinopass - 2,200 masl

1123 Figure 3. Co-ancestry matrix for figs (A: F. wassa; C: F. arfakensis) and their pollinating 1124 fig-wasps (from genera Kradibia and Ceratosolen: panels B and D respectively). The results show substantial population structure among the figs. They also reveal multiple wasp species responsible for pollination of the different fig populations. Values on the scale bar to the right of each matrix represent the estimated level of co-ancestry based on shared RAD loci. Highest levels of relatedness are indicated in dark blue. Lowest values are indicated in yellow. The population tree shows coalescent relationships between the populations, based on their inferred co-ancestry. Coloured boxes on the left indicate the collection site for each individual (legend on the bottom left). 


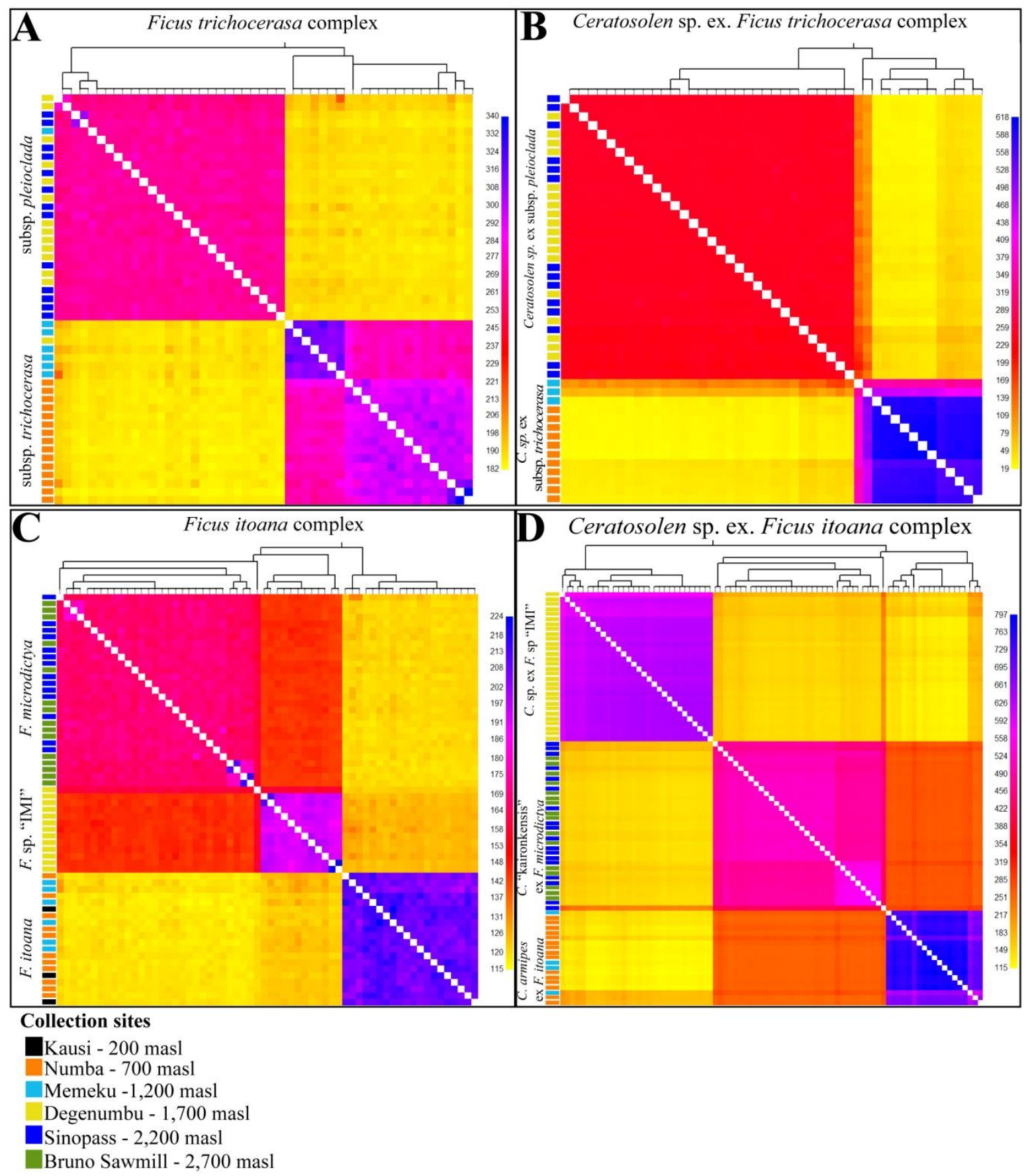

1133 Figure 4. Co-ancestry matrix for figs (A: F. trichocerasa; C: F. itoana complex) and their 1134 pollinating fig-wasps (panels $\mathrm{B}$ and $\mathrm{D}$ respectively). The results show clear clustering by 1135 (sub)species for both the figs and the wasps except for F. trichocerasa pollinators from the mid-elevations $(1,200-1,700 \mathrm{~m})$ where both species appear to converge in a contact zone. Values on the scale bar to the right of each matrix represent the estimated level of co-ancestry based on shared RAD loci. Highest levels of relatedness are indicated in dark blue. Lowest values are indicated in yellow. The population tree shows coalescent relationships between the populations, based on their inferred co-ancestry. Coloured boxes on the left indicate the collection site for each individual (legend on the bottom left). 


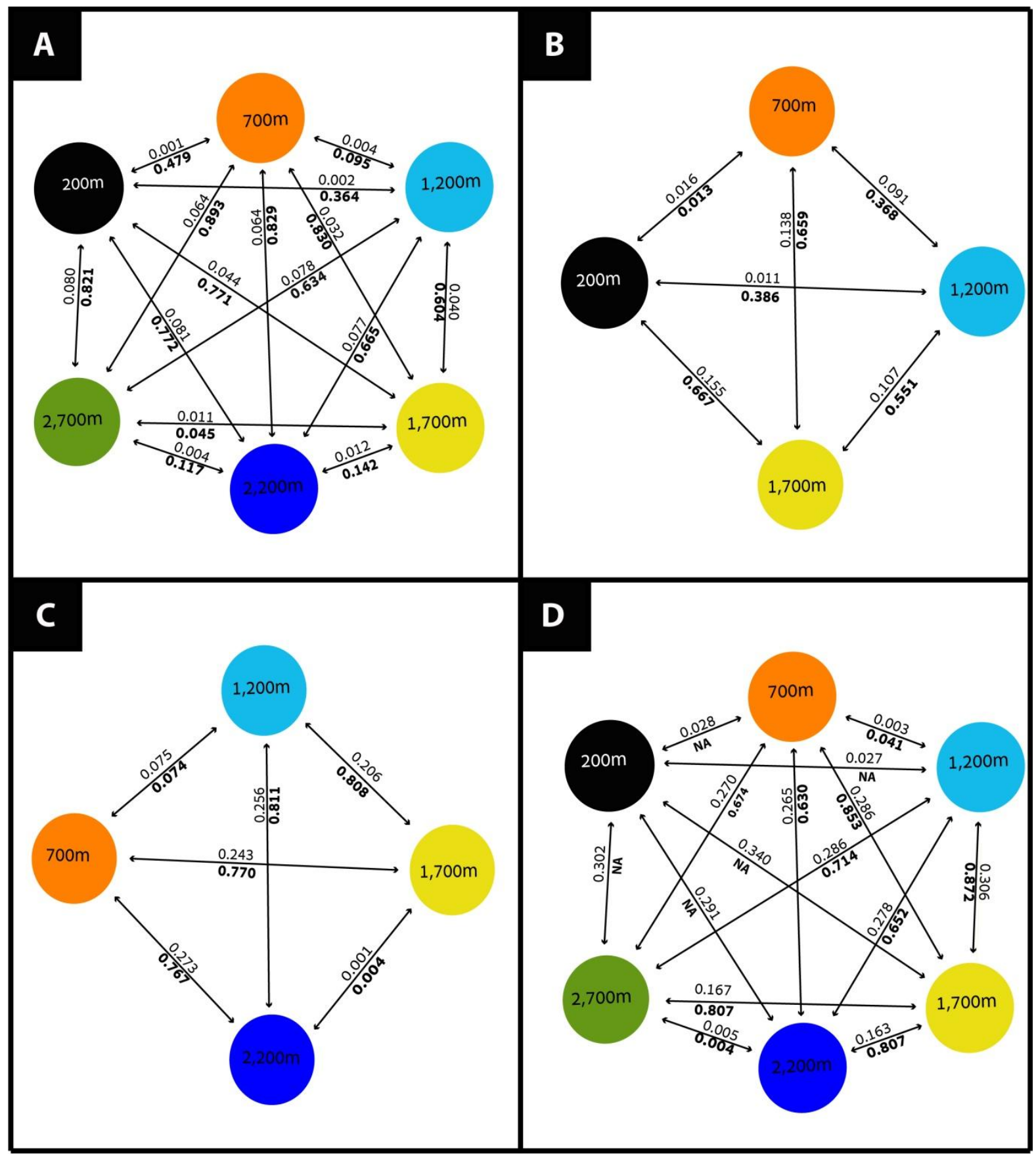

\section{Collection sites}

Kausi - 200 masl

Numba - 700 masl

Memeku -1,200 masl

Degenumbu - 1,700 masl

¿ Sinopass - 2,200 mas

1143 Figure 5. F F $_{\mathrm{ST}}$ comparisons between sites for $F$. wassa (A), F. arfakensis (B), $F$. trichocerasa

(C) and $F$. itoana complex (D) and their corresponding pollinating fig-wasps. Mean $\mathbf{F}_{\mathbf{S T}}$ values above the lines connecting the nodes (collection site) are for comparisons between figs while below the line in bold are mean $\mathbf{F}_{\mathbf{S T}}$ values for corresponding species of pollinating wasps. 